Contributions to Game Theory and Management, XIII, 218-243

\title{
Mechanism for Shifting Nash Equilibrium Trajectories to Cooperative Pareto Solutions in Dynamic Bimatrix Games *
}

\author{
Nikolay A. Krasovskii ${ }^{1}$ and Alexander M. Tarasyev ${ }^{1,2}$ \\ ${ }^{1}$ Krasovskii Institute of Mathematics and Mechanics UrB RAS, \\ S. Kovalevskoy Str., 16, Yekaterinburg, 620990, Russia \\ E-mail: nkrasovskiy@gmail.com \\ 2 Ural Federal University named after the first President of Russia B.N. Yeltsin, \\ Mira Str., 19, Yekaterinburg, 620002, Russia \\ E-mail: tam@imm.uran.ru, a.m.tarasyev@urfu.ru
}

\begin{abstract}
In the paper, constructions of the generalized method of characteristics are applied for calculating the generalized minimax (viscosity) solutions of Hamilton-Jacobi equations in dynamic bimatrix games. The structure of the game presumes interactions of two players in the framework of the evolutionary game model. Stochastic contacts between players occur according to the dynamic process, which can be interpreted as a system of Kolmogorov's differential equations with controls instead of probability parameters. It is assumed that control parameters are not fixed and can be constructed by the feedback principle. Two types of payoff functions are considered: short-term payoffs are determined in the current moments of time, and long-term payoffs are determined as limit functionals on the infinite time horizon. The notion of dynamic Nash equilibrium in the class of controlled feedbacks is considered for the long-term payoffs. In the framework of constructions of dynamic equilibrium, the solutions are designed on the basis of maximization of guaranteed payoffs. Such guaranteeing strategies are built in the framework of the theory of minimax (viscosity) solutions of Hamilton-Jacobi equations. The analytical formulas are obtained for the value functions in the cases of different orientations for the "zigzags" (broken lines) of acceptable situations in the static game. The equilibrium trajectories generated by the minimax solutions shift the system in the direction of cooperative Pareto points. The proposed approach provides new qualitative properties of the equilibrium trajectories in the dynamic bimatrix games which guarantee better results of payoffs for both players than static Nash equilibria. As an example, interactions of two firms on the market of innovative electronic devices are examined within the proposed approach for treating dynamic bimatrix games.
\end{abstract}

Keywords: optimal control, dynamic bimatrix games, value functions, minimax solutions of Hamilton-Jacobi equations, dynamic Nash equilibrium trajectories, shift to Pareto maximum.

\section{Introduction}

In the paper, a model of evolutionary non-zero sum game between two players (coalitions of players) is considered in the framework of differential games theory (Krasovskii and Krasovskii, 1995; Krasovskii, 1985; Krasovskii and Subbotin, 1988). We use some ideas and approaches proposed in (Kleimenov, 1993) for nonantagonistic differential games. The methods for analysis of evolutionary games

\footnotetext{
* The first author, Nikolay A. Krasovskii, is supported by the Russian Foundation for Basic Research (Project No. 18-01-00221a).
} 
suggested in the paper (Kryazhimskii and Osipov, 1995) are applied for derivation of the game dynamics. The main attention is paid to the construction of the dynamic Nash equilibrium with guaranteeing strategies of players, which maximize corresponding payoff functions (Pontryagin et al., 1961; Krasovskii and Subbotin, 1988). Solution trajectories are generated, which provide better result comparing to classic models, for example, models with the replicator dynamics.

The dynamics of game interactions corresponds to the differential games theory (Kleimenov, 1993; Krasovskii, 1985; Krasovskii and Subbotin, 1988; Kryazhimskii and Osipov, 1995; Kurzhanskii, 1977) and evolutionary game models (Basar and Olsder, 1982; Friedman, 1991; Hofbauer and Sigmund, 1988; Intriligator, 1971; Tarasyev, 1994).

The main idea of the paper is to apply the theory of generalized minimax (viscosity) solutions for Hamilton-Jacobi equations (Crandall and Lions, 1983; Subbotin, 1991) to analysis of the dynamic bimatrix games.

It is assumed that random interactions between players are presented by the controlled dynamic process, in which corresponding probabilities form a phase vector. Information signals for participants play the role of control parameters. Such dynamics can be interpreted as a generalization of the well-known Kolmogorov's equations, which are used in some stochastic models of mathematical economics and queue theory. The generalization is based on introduction of controls instead the probability parameters which describe incoming and outgoing flows inside groups of players. The dynamic process evolves on an infinite time interval. Participants' payoffs at each round are specified by the payoff matrices. Groups' payoffs are determined as the average value of participants' payoffs. Various types of these averages are considered: terminal (short run) - for a fixed terminal time, and multi-terminal (long run) - for the limit on the infinite time interval. Let us note that the non-zero sum game was analyzed in (Tarasyev, 1994) with the discounted integral payoff functionals. Multi-terminal functionals are associated with the concept which takes into the account not only local terminal interests of groups, but also are oriented on changes in the future.

The definition of the dynamic Nash equilibrium is introduced in the class of feedback control strategies. Let us note that feedbacks generated by the classic "punishing" strategy in static bimatrix games are a natural example of the Nash equilibrium in dynamic terms. The nature of such reactions are antagonistic: they minimize the payoff of the opponent, not maximizing their own.

A different approach is proposed on the basis of the concept of the "guarantee" providing better results than classic solutions. These new solutions are generated in the framework of the positional differential games theory and involve the guaranteeing feedbacks in the auxiliary zero-sum games (Kleimenov, 1993; Krasovskii and Subbotin, 1988; Petrosjan and Zenkevich, 2015). So, the non-zero sum games are considered in the framework of the theory for minimax solutions of HamiltonJacobi equations (Crandall and Lions, 1983; Subbotin, 1991; Subbotin and Tarasyev, 1985; Tarasyev, 1994). For the analytical construction of the value function and optimal guaranteeing feedbacks we use methods of the differential games theory (Kleimenov, 1993; Krasovskii and Subbotin, 1988), the generalized characteristics of the Hamilton-Jacobi equations (Subbotin, 1991), and constructions of the viability theory (Aubin, 1990). The corresponding necessary and sufficient conditions are verified for the value functions, particularly, in terms of conjugate derivatives 
(Subbotin and Tarasyev, 1985). The structure of the value functions is such that the synthesis of guaranteeing feedbacks for controls is determined by switching curves from one characteristic of the Hamilton-Jacobi equation to another one. Let us note, that similar ideas for the numerical construction of the solution are used in papers (Klaassen et al., 2004; Tarasyev and Watanabe, 2001; Krasovskii and Tarasyev, 2008; Krasovskii and Tarasyev, 2011; Krasovskii and Tarasyev, 2015; Krasovskii and Tarasyev, 2018).

The qualitative behavior of the proposed equilibrium solutions, generated by the guarantee synthesis, significantly differs from trajectories of evolutionary games presented in classic models with the dynamic replicator. Let us note that such trajectories usually converge to the static Nash equilibrium or circulate in its surroundings (Hofbauer and Sigmund, 1988). New equilibrium solutions are not smooth and have the switching regimes within characteristics of the Hamilton-Jacobi equations. Unlike classical trajectories, they are located at the intersection of areas, for which the values of players' payoffs are better than the corresponding payoffs calculated in the static Nash equilibrium. The proposed equilibrium solutions do not converge to the static Nash equilibrium, and their marginal payoffs are better than at the point of the Nash equilibrium. In this sense, one can say about shift of Nash equilibrium trajectories to the set of Pareto maximum points.

We provide analytic formulas for the value functions in the cases of different orientations for the "zigzags" (broken lines) of acceptable situations in the static game (Vorobyev, 1985). The equilibrium trajectories generated by the minimax solutions converge either to the intersection points of the synthesized switching lines, or to Pareto maximum points on the boundary of the square of acceptable situations, i.e. to "new" equilibrium points with better payoff indices. Thus, it is demonstrated that the proposed approach provides new qualitative properties of the equilibrium trajectories in the dynamic bimatrix games. As an example, we consider interactions of two firms on the market of innovative electronic devices, which give the dynamic bimatrix game with the left orientation of "zigzag" (broken line) of acceptable situations. We construct control synthesis for both players basing on the value functions as the generalized minimax solutions of the Hamilton-Jacobi equations, and generate the Nash equilibrium trajectories which shift the game solution in the direction of the Pareto maximum set.

\section{Evolutionary Game. Dynamic Nash Equilibrium}

\subsection{Model Dynamics, Payoff Functions}

Let us consider the system of differential equations, which describes the behavior dynamics of two players:

$$
\begin{aligned}
\dot{x}(t)=-x(t)+u(t), & x\left(t_{0}\right)=x_{0}, \\
\dot{y}(t)=-y(t)+v(t), & y\left(t_{0}\right)=y_{0} .
\end{aligned}
$$

Here the parameter $x=x(t), 0 \leq x \leq 1$ means the probability that the first player holds to the first strategy (respectively, $(1-x)$ is the probability that he holds to the second strategy). Parameter $y=y(t), 0 \leq y \leq 1$ stands for the probability of choosing the first strategy by the second player (respectively, $(1-y)$ is the probability that he holds to the second strategy). Control parameters $u=u(t)$ and $v=v(t)$ satisfy conditions $0 \leq u \leq 1,0 \leq v \leq 1$, and can be interpreted as signals that recommend changing strategies by players. 
For example, the value $u=0(v=0)$ corresponds to the signal: "change the first strategy to the second". The value $u=1(v=1)$ corresponds to the signal: "change the second strategy to the first". The value $u=x(v=y)$ corresponds to the signal: "keep the previous strategy".

Let us note, that the basis for the dynamics (1) and its properties were considered in (Kryazhimskii and Osipov, 1995; Tarasyev, 1994). In this dynamics, Kolmogorov's differential equations are generalized under the assumption that the coefficients of incoming and outgoing flows between players are not set a priori and can be constructed in the control process on the feedback principle.

As an interpretation of the dynamics (1), we consider the game interaction of two players (or their investments) on two markets. Let $x$ be a part of the funds, which the first player (it may be a financial or industrial group) invest in the first market (this may be a financial market, a market of goods or innovative technologies (Vorobyev, 1985)). Respectively, $1-x$ is the investment of the first player in the second market. Let $y$ be the part of the funds that the second player invest in the first market. Respectively, $1-y$ is the investment of the second player in the second market. Let us assume that activity of players is regulated by a governing body. Using control parameters $u$ and $v$, a governing body can influence on the distribution of funds $x$ and $y$. The dynamics of this influence is described by the system (1) and provides some inertia of players in relation to control signals $u, v$, while the velocities $\dot{x}, \dot{y}$ of changing of capital proportions $x, y$ are not right proportional to the signals, and depend on the size of these proportions. For example, the first equation in (1) means that according to the signal $u=0$ the proportion $x$ decreases to zero according to the dynamics $x(t)=-x(t)$.

An important property of dynamics $(1)$ is that the square, $(x, y) \in[0,1] \times[0,1]$, is its strongly invariant set. That is, any trajectory of dynamics (1), which starts in the square, survives in it on the infinite time horizon.

Let us assume, that payoffs of the first (second) player are described by the matrix $A=a_{i j}\left(B=b_{i j}\right)$

$$
A=\left(\begin{array}{ll}
a_{11} & a_{12} \\
a_{21} & a_{22}
\end{array}\right), \quad B=\left(\begin{array}{ll}
b_{11} & b_{12} \\
b_{21} & b_{22}
\end{array}\right) .
$$

Let us consider, for example, for the game of two players on two markets the following situation, which we will call almost antagonistic. Let us assume that the first market is more profitable for investments than the second one. The first player is stronger than the the second one. He is trying to capture both markets. The second player is trying to avoid interactions with the first player on the same market.

The terminal payoff functions of players are determined as a mathematical expectation of payoffs generated by corresponding matrices $A, B$, and can be interpreted as "local" interests of players

$$
\begin{gathered}
g_{A}(x(T), y(T))= \\
a_{11} x(T) y(T)+a_{12} x(T)(1-y(T))+a_{21}(1-x(T)) y(T)+a_{22}(1-x(T))(1-y(T))= \\
C_{A} x(T) y(T)-\alpha_{1} x(T)-\alpha_{2} y(T)+a_{22} .
\end{gathered}
$$

The function $g_{B}$ for the matrix $B$ is determined analogously. Here the coefficients $C_{A}, \alpha_{1}, \alpha_{2}$ are determined according to the classical theory of bimatrix games 
(Vorobyev, 1985)

$$
C_{A}=a_{11}-a_{12}-a_{21}+a_{22}, \quad \alpha_{1}=a_{22}-a_{12}, \quad \alpha_{2}=a_{22}-a_{21} .
$$

The coefficients $C_{B}, \beta_{1}, \beta_{2}$ for the matrix $B$ are determined analogously.

The "global" interests $J_{A}^{\infty}$ of the first player are determined as multivalued (twodigit) functions formed by lower and upper limits of average values

$$
\begin{aligned}
& J_{A}^{\infty}=\left[J_{A}^{-}, J_{A}^{+}\right], \\
& J_{A}^{-}=J_{A}^{-}(x(\cdot), y(\cdot))=\liminf _{t \rightarrow \infty} g_{A}(x(t), y(t)), \\
& J_{A}^{+}=J_{A}^{+}(x(\cdot), y(\cdot))=\limsup _{t \rightarrow \infty} g_{A}(x(t), y(t)),
\end{aligned}
$$

calculated for the trajectories $(x(\cdot), y(\cdot))$ of the system (1). For the second player the "global" interests $J_{B}^{\infty}$ are determined symmetrically.

Let us consider an evolutionary non-zero sum game with the dynamics (1) and global payoffs, given by the scheme (3). In the differential games theory (Kleimenov, 1993; Krasovskii and Subbotin, 1988) there exists an approach for construction of the equilibrium solutions from the class of strategies, defined on the feedback principle $U=u(t, x, y, \varepsilon), V=v(t, x, y, \varepsilon)$, for the non-zero sum games. Such approach is based on the solution of auxiliary zero sum games. According to our statement of the problem, we consider zero sum games for the functionals $J_{A}^{-}, J_{A}^{+}, J_{B}^{-}, J_{B}^{+}$. It is known, that problems with zero sum can be solved in the framework of differential games theory, and the solution for guaranteeing strategies can be obtained in the framework of the dynamic programming principle. Such principle requires finding the value functions, which are generalized minimax solutions of HamiltonJacobi equations, namely, the problem is reduced to solving the equations in partial derivatives of the first order.

\subsection{Dynamic Nash Equilibrium}

Following (Kleimenov, 1993; Kryazhimskii and Osipov, 1995), we present the definition of the dynamic Nash equilibrium in the class of positional strategies (feedbacks) $U=u(t, x, y, \varepsilon), V=v(t, x, y, \varepsilon)$ for the non-zero sum game with the given dynamics and the multivalued payoff functionals.

Definition 1. Let $\varepsilon>0$ and $\left(x_{0}, y_{0}\right) \in[0,1] \times[0,1]$. The pair of feedbacks $U^{0}=$ $u^{0}(t, x, y, \varepsilon), V^{0}=v^{0}(t, x, y, \varepsilon)$ is called the Nash equilibrium at the initial point $\left(x_{0}, y_{0}\right)$, if for any other feedbacks $U=u(t, x, y, \varepsilon), V=v(t, x, y, \varepsilon)$ the following conditions take place: for any trajectories

$$
\begin{gathered}
\left(x^{0}(\cdot), y^{0}(\cdot)\right) \in X\left(x_{0}, y_{0}, U^{0}, V^{0}\right), \\
\left(x_{1}(\cdot), y_{1}(\cdot)\right) \in X\left(x_{0}, y_{0}, U, V^{0}\right),\left(x_{2}(\cdot), y_{2}(\cdot)\right) \in X\left(x_{0}, y_{0}, U^{0}, V\right)
\end{gathered}
$$

the inequalities are true:

$$
J_{A}^{-}\left(x^{0}(\cdot), y^{0}(\cdot)\right) \geq J_{A}^{+}\left(x_{1}(\cdot), y_{1}(\cdot)\right)-\varepsilon, \quad J_{B}^{-}\left(x^{0}(\cdot), y^{0}(\cdot)\right) \geq J_{B}^{+}\left(x_{2}(\cdot), y_{2}(\cdot)\right)-\varepsilon .
$$

Here the symbol $X$ stands for the set of trajectories, which start from the initial point $\left(x_{0}, y_{0}\right)$ and are generated by the corresponded strategies $\left(U^{0}, V^{0}\right),\left(U, V^{0}\right)$, $\left(U^{0}, V\right)$ (see (Krasovskii and Subbotin, 1988)). 


\subsection{Auxiliary Zero-Sum Games}

For construction of the desired equilibrium feedbacks $U^{0}, V^{0}$ we use the results (Kleimenov, 1993). According to this approach, we construct the equilibrium using optimal feedbacks for differential games $\Gamma_{A}=\Gamma_{A}^{-} \cup \Gamma_{A}^{+}$and $\Gamma_{B}=\Gamma_{B}^{-} \cup \Gamma_{B}^{+}$ with payoffs $J_{A}^{\infty}$ and $J_{B}^{\infty}$. In the game $\Gamma_{A}$ the first player maximizes the functional $J_{A}^{-}(x(\cdot), y(\cdot))$ with the guarantee, using the feedback $U=u(t, x, y, \varepsilon)$, and the second player, on the contrary, tries to minimize the functional $J_{A}^{+}(x(\cdot), y(\cdot))$, using the feedback $V=v(t, x, y, \varepsilon)$. Vice versa, in the game $\Gamma_{B}$ the second player maximizes the functional $J_{B}^{-}(x(\cdot), y(\cdot))$ with the guarantee, and the first player minimizes the functional $J_{B}^{+}(x(\cdot), y(\cdot))$.

Let us introduce the following notations. The feedbacks solving, respectively, the problem of guaranteeing maximization of the payoff functionals $J_{A}^{-}, J_{B}^{-}$are denoted by symbols $u_{A}^{0}=u_{A}^{0}(t, x, y, \varepsilon)$ and $v_{B}^{0}=v_{B}^{0}(t, x, y, \varepsilon)$. Let us note, that such feedbacks present the guaranteeing maximization of players' payoffs in the long run, and can be called "positive" feedbacks. By symbols $u_{B}^{0}=u_{B}^{0}(t, x, y, \varepsilon)$ and $v_{A}^{0}=v_{A}^{0}(t, x, y, \varepsilon)$ we denote feedbacks which are most unfavorable to the opposing players; namely, those feedbacks, which minimizes the payoff functional $J_{B}^{+}, J_{A}^{+}$of opposing players, respectively. Let us call these feedbacks "punishing".

Let us note, that inflexible solutions to these problems can be obtained in the framework of the classical theory of bimatrix games. In fact, let us suppose for definiteness (although this is not essential for constructing solutions in the general case) that the following conditions are satisfied:

$$
\begin{aligned}
& C_{A}>0, \quad 0<x_{A}=\frac{\alpha_{2}}{C_{A}}<1, \quad 0<y_{A}=\frac{\alpha_{1}}{C_{A}}<1, \\
& C_{B}<0, \quad 0<x_{B}=\frac{\beta_{2}}{C_{B}}<1, \quad 0<y_{B}=\frac{\beta_{1}}{C_{B}}<1 .
\end{aligned}
$$

Proposition 1. The differential games $\Gamma_{A}^{-}, \Gamma_{A}^{+}\left(\Gamma_{B}^{-}, \Gamma_{B}^{+}\right)$have equal values

$$
\omega_{A}^{-}=\omega_{A}^{+}=\omega_{A}=\frac{a_{22} C_{A}-\alpha_{1} \alpha_{2}}{C_{A}}, \quad \omega_{B}^{-}=\omega_{B}^{+}=\omega_{B}=\frac{b_{22} C_{B}-\beta_{1} \beta_{2}}{C_{B}}
$$

for arbitrary initial position $\left(x_{0}, y_{0}\right) \in[0,1] \times[1,0]$. These values can be guaranteed by "positive" feedbacks $u_{A}^{\mathrm{cl}}, v_{B}^{\mathrm{cl}}$ corresponding to the classic solutions $x_{A}, y_{B}$

$$
u_{A}^{\mathrm{cl}}(x, y)=\left\{\begin{array}{l}
0, \quad x_{A}<x \leq 1 \\
1, \quad 0 \leq x<x_{A} \\
{[0,1], \quad x=x_{A}}
\end{array}, \quad v_{B}^{\mathrm{cl}}(x, y)=\left\{\begin{array}{l}
0, \quad y_{B}<y \leq 1 \\
1, \quad 0 \leq y<y_{B} \\
{[0,1], \quad y=y_{B}}
\end{array}\right.\right.
$$

The "punishing" feedbacks are determined by formulas

$$
u_{B}^{\mathrm{cl}}(x, y)=\left\{\begin{array}{l}
0, \quad x_{B}<x \leq 1 \\
1, \quad 0 \leq x<x_{B} \\
{[0,1], \quad x=x_{B}}
\end{array}, \quad v_{A}^{\mathrm{cl}}(x, y)=\left\{\begin{array}{l}
0, \quad y_{A}<y \leq 1 \\
1, \quad 0 \leq y<y_{A} \\
{[0,1], \quad y=y_{A}}
\end{array}\right.\right.
$$

and correspond to the classic static solutions $x_{B}, y_{A}$, which generate the static Nash equilibrium $N E=\left(x_{B}, y_{A}\right)$.

Proof. The proof of this proposition is justified by the direct substitution of strategies $u_{A}^{\mathrm{cl}}, v_{B}^{\mathrm{cl}}$ and strategies $u_{B}^{\mathrm{cl}}, v_{A}^{\mathrm{cl}}$ in the dynamics (1). 
Note 1. The values of payoff functions $g_{A}(x, y), g_{B}(x, y)$ coincide at points $\left(x_{A}, y_{B}\right)$, $\left(x_{B}, y_{A}\right)$

$$
g_{A}\left(x_{A}, y_{B}\right)=g_{A}\left(x_{B}, y_{A}\right)=\omega_{A}, \quad g_{B}\left(x_{A}, y_{B}\right)=g_{B}\left(x_{B}, y_{A}\right)=\omega_{B} .
$$

The point $N E=\left(x_{B}, y_{A}\right)$ is the "mutually punishing" Nash equilibrium, and the point $x_{A}, y_{B}$ does not possess the equilibrium properties in the corresponding static game.

\subsection{Construction of the Nash Equilibrium}

Let us construct the pair of feedbacks, which form the dynamic Nash equilibrium. For that, we connect the "positive" feedbacks $u_{A}^{0}, v_{B}^{0}$ and the "punishing" feedbacks $u_{B}^{0}, v_{A}^{0}$.

Let us choose the initial position $\left(x_{0}, y_{0}\right) \in[0,1] \times[0,1]$ and the accuracy parameter $\varepsilon>0$. Let us choose the trajectory $\left(x^{0}(\cdot), y^{0}(\cdot)\right) \in X\left(x_{0}, y_{0}, U_{A}^{0}(\cdot), v_{B}^{0}(\cdot)\right)$, generated by the "positive" feedbacks $u_{A}^{0}=U_{A}^{0}(t, x, y, \varepsilon)$ and $v_{B}^{0}=v_{B}^{0}(t, x, y, \varepsilon)$. Let us take the time moment $T_{\varepsilon}>0$ such that

$$
\begin{gathered}
g_{A}\left(x^{0}(t), y^{0}(t)\right)>J_{A}^{-}\left(x^{0}(\cdot), y^{0}(\cdot)\right)-\varepsilon, \quad g_{B}\left(x^{0}(t), y^{0}(t)\right)>J_{B}^{-}\left(x^{0}(\cdot), y^{0}(\cdot)\right)-\varepsilon, \\
t \in\left[T_{\varepsilon},+\infty\right] .
\end{gathered}
$$

Let us denote by $u_{A}^{\varepsilon}(t):\left[0, T_{\varepsilon}\right) \rightarrow[0,1], v_{B}^{\varepsilon}(t):\left[0, T_{\varepsilon}\right) \rightarrow[0,1]$ the step-bystep realization of the strategies $v_{A}^{0}, v_{B}^{0}$ such that the corresponding step-by-step mechanism $\left(x_{\varepsilon}(\cdot), y_{\varepsilon}(\cdot)\right)$ satisfies the condition

$$
\max _{t \in\left[0, T_{\varepsilon}\right]}\left\|\left(x^{0}(t), y^{0}(t)\right)-\left(x_{\varepsilon}(t), y_{\varepsilon}(t)\right)\right\|<\varepsilon .
$$

From the results of the paper (Kleimenov, 1993) the next proposition follows.

Proposition 2. The pair of feedbacks $U^{0}=u^{0}(t, x, y, \varepsilon), V^{0}=v^{0}(t, x, y, \varepsilon)$, connecting together the "positive" feedbacks $u_{A}^{0}, v_{B}^{0}$ and the "punishing" feedbacks $u_{B}^{0}$, $v_{A}^{0}$ according to the relations

$$
\begin{aligned}
U^{0} & =\left\{\begin{array}{l}
u_{A}^{\varepsilon}(t), \text { if }\left\|(x, y)-\left(x_{\varepsilon}(t), y_{\varepsilon}(t)\right)\right\|<\varepsilon, \\
u_{B}^{0}(x, y), \text { otherwise, }
\end{array}\right. \\
V^{0} & =\left\{\begin{array}{l}
v_{B}^{\varepsilon}(t), \text { if }\left\|(x, y)-\left(x_{\varepsilon}(t), y_{\varepsilon}(t)\right)\right\|<\varepsilon, \\
v_{A}^{0}(x, y), \text { otherwise, }
\end{array}\right.
\end{aligned}
$$

is the dynamic $\varepsilon$-Nash equilibrium.

Note 2. Let us note that the number $\varepsilon$ can be interpreted as the parameter of "trust" of players to each other or as the level of "risk", which players allow in the game. This parameter determines the risk barrier that surrounds the equilibrium trajectory $\left(x_{\varepsilon}(\cdot), y_{\varepsilon}(\cdot)\right)$. The players either follow the equilibrium trajectory, without leaving the prescribed risk barrier, getting the more profitable index of values, or stepping over it and the "punishing" strategy gives worse results.

Below we construct the flexible "positive" feedbacks, which generate trajectories $\left(x^{\mathrm{f}}(\cdot), y^{\mathrm{fl}}(\cdot)\right)$ reducing to "better" positions, than the inflexible dynamic equilibrium $\left(x_{B}, y_{A}\right),\left(x_{A}, y_{B}\right)$ by both criteria $J_{A}^{\infty}\left(x^{\mathrm{f}}(\cdot), y^{\mathrm{f}}(\cdot)\right) \geq \omega_{A}, J_{B}^{\infty}\left(x^{\mathrm{fl}}(\cdot), y^{\mathrm{fl}}(\cdot)\right) \geq \omega_{B}$. 


\section{Differential Game with Terminal Functional}

\subsection{Value Functions and Generalized Solutions of Hamilton-Jacobi Equations}

In this section, we consider an auxiliary terminal differential zero-sum game with the dynamics (1) and payoff functionals (2). Further, solutions of terminal differential games are used for the construction of anticipating feedbacks by calculating the lower envelope of multi-terminal functionals. The value functions $w_{i}(T, t, x, y)$, $i=1,2$ of terminal games are determined as values of corresponding maximins (minimaxs)

$$
\begin{gathered}
w_{1}\left(T, t_{0}, x_{0}, y_{0}\right)= \\
\max _{u(t, x, y)} \min _{\left(x_{1}(\cdot), y_{1}(\cdot)\right)} g_{A}\left(x_{1}(T), y_{1}(T)\right)=\min _{v(t, x, y)\left(x_{2}(\cdot), y_{2}(\cdot)\right)} \\
w_{2}\left(T, t_{0}, x_{0}, y_{0}\right)= \\
\max _{v(t, x, y)\left(x_{2}(\cdot), y_{2}(\cdot)\right)} g_{B}\left(x_{2}(T), y_{2}(T)\right), \\
\min _{u(t, x, y)\left(x_{1}(\cdot), y_{1}(\cdot)\right)} g_{B}\left(x_{1}(T), y_{1}(T)\right)
\end{gathered}
$$

for each initial position $\left(t_{0}, x_{0}, y_{0}\right)$. Here trajectories $\left(x_{1}(\cdot), y_{1}(\cdot)\right)$ are generated by feedbacks $u(t, x, y, \varepsilon)$ and random behaviors $v(t)$. Trajectories $\left(x_{2}(\cdot), y_{2}(\cdot)\right)$ are generated by feedbacks $v(t, x, y, \varepsilon)$ and random behaviors $u(t)$ from the initial position $\left(t_{0}, x_{0}, y_{0}\right)$.

The value functions $w_{i}(T, t, x, y), i=1,2$ satisfy the principle of the dynamic programming, which implies the existence of non-increasing and non-decreasing directions achievable for the dynamic system at each current position (the so called properties $u$ and $v$ of stability of the value function). At the points, where the value functions are differentiable, these properties pass into the first-order partial differential Hamilton-Jacobi equations

$$
\begin{aligned}
& \frac{\partial w_{1}}{\partial t}-\frac{\partial w_{1}}{\partial x} x-\frac{\partial w_{1}}{\partial y} y+\max _{0 \leq u \leq 1} \frac{\partial w_{1}}{\partial x} u+\min _{0 \leq v \leq 1} \frac{\partial w_{1}}{\partial y} v=0 \\
& \frac{\partial w_{2}}{\partial t}-\frac{\partial w_{2}}{\partial x} x-\frac{\partial w_{2}}{\partial y} y+\min _{0 \leq u \leq 1} \frac{\partial w_{2}}{\partial x} u+\max _{0 \leq v \leq 1} \frac{\partial w_{2}}{\partial y} v=0 .
\end{aligned}
$$

The value functions $w_{i}(T, t, x, y), i=1,2$ also satisfy the boundary condition, when $t=T$ :

$$
w_{1}(T, T, x, y)=g_{A}(x, y), \quad w_{2}(T, T, x, y)=g_{B}(x, y)
$$

Let us consider the terminal boundary problems (4)-(6) for the value functions $w_{1}(T, t, x, y), w_{2}(T, t, x, y)$. It is known (Crandall and Lions, 1983; Subbotin, 1991), that value function $w_{1}(T, t, x, y)$ coincides with the generalized solution of this problem, which is unique and is determined by the terminal boundary value (6), and the pair of differential inequalities for conjugate derivatives $D^{*} w_{1}$ and $D_{*} w_{1}$, corresponding to the Hamilton-Jacobi equation (4)

$$
\begin{gathered}
D^{*} w_{1}(T, t, x, y)\left|(s) \geq H(x, y, s), \quad D_{*} w_{1}(T, t, x, y)\right|(s) \leq H(x, y, s), \\
(t, x, y) \in\left[t_{0}, T\right] \times(0,1) \times(0,1), \quad s=\left(s_{1}, s_{2}\right) \in \mathbb{R}^{2}
\end{gathered}
$$


The conjugate derivatives $D^{*} w_{1}$ and $D_{*} w_{1}$ and the Hamiltonian $H$ are given by the formulas (Subbotin and Tarasyev, 1985)

$$
\begin{gathered}
D^{*} w_{1}(T, t, x, y) \mid(s)=\sup _{h \in \mathbb{R}^{2}}\left(\langle s, h\rangle-\partial_{-} w_{1}(T, t, x, y) \mid(1, h)\right), \\
D_{*} w_{1}(T, t, x, y) \mid(s)=\inf _{h \in \mathbb{R}^{2}}\left(\langle s, h\rangle-\partial_{+} w_{1}(T, t, x, y) \mid(1, h)\right), \\
H(x, y, s)=-s_{1} x-s_{2} y+\max _{0 \leq u \leq 1} s_{1} u+\min _{0 \leq v \leq 1} s_{2} v .
\end{gathered}
$$

Expressions $\partial_{-} w_{1}(T, t, x, y)\left|(1, h), \partial_{+} w_{1}(T, t, x, y)\right|(1, h)$ stand for directional derivatives of the value function $w_{1}$ at the point $(t, x, y)$ in the direction $(1, h), h=$ $\left(h_{1}, h_{2}\right) \in \mathbb{R}^{2}$

$$
\begin{aligned}
& \left.\partial_{-} w_{1}(T, t, x, y) \mid(1, h)\right)=\liminf _{\delta \downarrow 0} \frac{w_{1}\left(T, t+\delta, x+\delta h_{1}, y+\delta h_{2}\right)-w_{1}(T, t, x, y)}{\delta}, \\
& \left.\partial_{+} w_{1}(T, t, x, y) \mid(1, h)\right)=\limsup _{\delta \downarrow 0} \frac{w_{1}\left(T, t+\delta, x+\delta h_{1}, y+\delta h_{2}\right)-w_{1}(T, t, x, y)}{\delta} .
\end{aligned}
$$

For the piecewise smooth value function $w_{1}$ the directional derivatives and the conjugate derivatives can be calculated in the framework of non-smooth and convex analysis. Let us assume that in the vicinity $(t, x, y) \in O_{\varepsilon}\left(t_{*}, x_{*}, y_{*}\right)$ the function $w_{1}$ is given by the formulas

$$
w_{1}(T, t, x, y)=\min _{i \in I} \max _{j \in J} \varphi_{i j}(T, t, x, y)=\max _{j \in J} \min _{i \in I} \varphi_{i j}(T, t, x, y) .
$$

Here symbols $I, J$ stand for the finite sets of indices $i, j$ respectively.

The directional derivatives in this case are determined by the relations

$$
\begin{gathered}
\partial w_{1}\left(T, t_{*}, x_{*}, y_{*}\right) \mid(h)=\min _{i \in I} \max _{j \in J}\left(a_{i j}+\left\langle b_{i j}, h\right\rangle\right)=\max _{j \in J} \min _{i \in I}\left(a_{i j}+\left\langle b_{i j}, h\right\rangle\right), \\
a_{i j}=\frac{\partial \varphi_{i j}}{\partial t}, \quad b_{i j}=\left(\frac{\partial \varphi_{i j}}{\partial x}, \frac{\partial \varphi_{i j}}{\partial y}\right) .
\end{gathered}
$$

Let us introduce the notations

$$
C=\bigcap_{i \in I} B_{i}, \quad B_{i}=\operatorname{co}\left\{b_{i j}: j \in J\right\}, \quad D=\bigcap_{j \in J} B_{j}, \quad B_{j}=\operatorname{co}\left\{b_{i j}: i \in I\right\} .
$$

The conjugate derivatives are determined by the relations

$$
\begin{aligned}
& D^{*} w_{1}\left(T, t_{*}, x_{*}, y_{*}\right) \mid(s)= \begin{cases}\max _{i \in I} \min \left\{-\sum_{j \in J} \lambda_{j}(s) a_{i j}\right\}, & s \in C, \\
+\infty, & s \notin C,\end{cases} \\
& D_{*} w_{1}\left(T, t_{*}, x_{*}, y_{*}\right) \mid(s)= \begin{cases}\min _{j \in J} \max \left\{-\sum_{i \in I} \lambda_{i}(s) a_{i j}\right\}, & s \in D, \\
-\infty, & s \notin D .\end{cases}
\end{aligned}
$$

Here the coefficients $\lambda_{j}(s)$ satisfy the conditions

$$
\sum_{j \in J} \lambda_{j}(s) b_{i j}=s, \quad \lambda_{j}(s) \geq 0, \quad \sum_{j \in J} \lambda_{j}(s)=1 .
$$


Mechanism for Shifting Nash Equilibrium Trajectories to Cooperative Solutions 227

\subsection{Description of Analytical Solution for Boundary Problem}

The boundary problem (4), (6) has an analytical solution. The corresponding value function $w_{1}(T, t, x, y)$ is piecewise smooth and consists of five smooth functions $\varphi_{k}(T, t, x, y), k=1, \ldots, 5$. The analytical formulas for smooth components $\varphi_{k}(T, t, x, y), k=1, \ldots, 5$ can be obtained using the method of characteristics for the linear Hamilton-Jacobi equations, which arise from nonlinear (4) by the substituting different combinations of the extremal values 0 and 1 in the expressions max and min. Let us provide the formulas for these functions:

$$
\begin{gathered}
\varphi_{1}(T, t, x, y)=C_{A} e^{2(t-T)} x y-\alpha_{1} e^{t-T} x-\alpha_{2} e^{t-T} y+a_{22} \\
\varphi_{2}(T, t, x, y)=C_{A} e^{2(t-T)} x y-\alpha_{1} e^{t-T} x \\
-\left(C_{A} e^{2(t-T)}+\left(\alpha_{2}-C_{A}\right) e^{(t-T)}\right) y+\alpha_{1} e^{(t-T)}+a_{12} \\
\varphi_{3}(T, t, x, y)=C_{A} e^{2(t-T)} x y-\left(C_{A} e^{2(t-T)}+\left(\alpha_{1}-C_{A}\right) e^{(t-T)}\right) x- \\
\left(C_{A} e^{2(t-T)}+\left(\alpha_{2}-C_{A}\right) e^{(t-T)}\right) y+C_{A} e^{2(t-T)}+\left(\alpha_{1}+\alpha_{2}-2 C_{A}\right) e^{(t-T)}+a_{11}, \\
\varphi_{4}(T, t, x, y)=C_{A} e^{2(t-T)} x y-\left(C_{A} e^{2(t-T)}+\left(\alpha_{1}-C_{A}\right) e^{(t-T)}\right) x- \\
\alpha_{2} e^{t-T} y+\alpha_{2} e^{t-T}+a_{21}, \\
\varphi_{5}(T, t, x, y)=\frac{a_{22} C_{A}-\alpha_{1} \alpha_{2}}{C_{A}}=\frac{a_{11} a_{22}-a_{12} a_{21}}{C_{A}}=\frac{D_{A}}{C_{A}}=v_{A} .
\end{gathered}
$$

The functions $\varphi_{k}, k=1, \ldots, 5$ are pasting together on the four lines $L_{m}=L_{m}(T, t)$, $m=1, \ldots, 4$

$$
\begin{array}{ll}
L_{1}=\{(x, y): & \left.x_{1}(T, t) \leq x \leq 1, \quad y=y_{2}(T, t)\right\}, \\
L_{2}=\{(x, y): & \left.x=x_{1}(T, t), \quad y_{1}(T, t) \leq y \leq 1\right\}, \\
L_{3}=\left\{(x, y): \quad 0 \leq x \leq x_{2}(T, t), \quad y=y_{1}(T, t)\right\}, \\
L_{4}=\left\{(x, y): \quad x=x_{2}(T, t), \quad 0 \leq y \leq y_{2}(T, t)\right\} .
\end{array}
$$

Here

$$
\begin{array}{ll}
x_{1}(T, t)=\max \left\{0,1-\left(1-\frac{\alpha_{2}}{C_{A}}\right) e^{(T-t)}\right\}, & x_{2}(T, t)=\max \left\{1, \frac{\alpha_{2}}{C_{A}} e^{(T-t)}\right\}, \\
y_{1}(T, t)=\max \left\{0,1-\left(1-\frac{\alpha_{1}}{C_{A}}\right) e^{(T-t)}\right\}, & y_{2}(T, t)=\max \left\{1, \frac{\alpha_{1}}{C_{A}} e^{(T-t)}\right\} .
\end{array}
$$

Proposition 3. The value function $w_{1}(T, t, x, y)$ is determined by the relation

$$
w_{1}(T, t, x, y)=\varphi_{k}(T, t, x, y), \quad(x, y) \in D_{k}(T, t), \quad k=1, \ldots, 5 .
$$

Here domains $D_{k}=D_{k}(T, t), k=1, \ldots, 5$ are given by inequalities

$$
\begin{aligned}
& D_{1}(T, t)=\left\{(x, y) \in[0,1] \times[0,1]: \quad x_{2}(T, t) \leq x \leq 1, \quad 0 \leq y \leq y_{2}(T, t)\right\}, \\
& D_{2}(T, t)=\left\{(x, y) \in[0,1] \times[0,1]: \quad x_{1}(T, t) \leq x \leq 1, \quad y_{2}(T, t) \leq y \leq 1\right\}, \\
& D_{3}(T, t)=\left\{(x, y) \in[0,1] \times[0,1]: \quad 0 \leq x \leq x_{1}(T, t), \quad y_{1} \leq y \leq 1(T, t)\right\}, \\
& D_{4}(T, t)=\left\{(x, y) \in[0,1] \times[0,1]: \quad 0 \leq x \leq x_{2}(T, t), \quad 0 \leq y \leq y_{1}(T, t)\right\}, \\
& D_{5}(T, t)=\left\{(x, y) \in[0,1] \times[0,1]: \quad x_{1}(T, t) \leq x \leq x_{2}(T, t),\right. \\
& \left.y_{1}(T, t) \leq y \leq y_{2}(T, t)\right\} \text {. }
\end{aligned}
$$


One can check the validity of differential inequalities in the necessary and sufficient conditions $(6),(7)$ for the function $w_{1}(T, t, x, y)$, determined by the formulas (12), (13).

\section{Value Function of the Game with Multi-Terminal Payoff Functional}

\subsection{Differential Game with Multi-terminal Functional}

In the previous section we obtained the solution for the auxiliary terminal boundary problem (4), (6). The solution of this problem (the value function) $w_{1}(T, t, x, y)$ depends on the terminal time moment $T$. Definitely, such solution is not a proper one in the evolutionary sense, because we obtain a "good" result only at the time moment $T$, but not in other time moments, including infinity. Thus, here we construct the value function for the differential game with the multi-terminal payoff functional

$$
G_{A}(x(\cdot), y(\cdot))=\inf _{t_{0} \leq t<+\infty} g_{A}(x(t), y(t)) .
$$

The functional (14) determines the foreseeing principle, since it takes into the account future positions $g_{A}(x(t), y(t))$, starting at the initial time $t_{0}$, and ending at infinity $+\infty$.

Using results obtained in the differential games theory (Krasovskii and Subbotin, 1988) and the viability theory (Aubin, 1990), one can prove that the differential zero-sum game with the dynamics (1) and the functional (14) has the value. More precisely, the next statement is true.

Theorem 1. There exists the saddle point that determines the stationary value function

$$
\begin{array}{r}
\sup _{u(t, x, y, \varepsilon)} \inf _{\left(x_{1}(\cdot), y_{1}(\cdot)\right)} \inf _{s \in\left[t_{0},+\infty\right]} g_{A}\left(x_{1}(s), y_{1}(s)\right)= \\
\inf _{v(t, x, y, \varepsilon)} \sup _{s \in\left[x_{2}(\cdot), y_{2}(\cdot)\right)} \inf _{s \in[+\infty} g_{A}\left(x_{2}(s), y_{2}(s)\right)= \\
\lim _{T \rightarrow+\infty} \min _{v(t, x, y, \varepsilon)} \max _{\left(x_{2}(\cdot), y_{2}(\cdot)\right)} \min _{s \in\left[t_{0}, T\right]} g_{A}\left(x_{2}(s), y_{2}(s)\right)= \\
\lim _{T \rightarrow+\infty} \max _{u(t, x, y, \varepsilon)} \min _{\left(x_{1}(\cdot), y_{1}(\cdot)\right)} \min _{s \in\left[t_{0}, T\right]} g_{A}\left(x_{1}(s), y_{1}(s)\right)=w_{A}\left(t_{0}, x_{0}, y_{0}\right)=w_{A}\left(x_{0}, y_{0}\right) .
\end{array}
$$

Here the trajectories $\left(x_{1}(\cdot), y_{1}(\cdot)\right),\left(x_{2}(\cdot), y_{2}(\cdot)\right)$ are generated from the initial position $\left(t_{0}, x_{0}, y_{0}\right)$ by feedbacks $u(t, x, y, \varepsilon), v(t, x, y, \varepsilon)$ of the maximizing and minimizing players, respectively, and arbitrary controls of their opponents.

Proof. The proof follows from the theorem on the alternative (Krasovskii and Subbotin, 1988), the stationary property of the dynamics (1), the finiteness of values of the functional $G_{A}(14)$, and can be deduced through the concept of the core viability (Aubin, 1990). The scheme of the proof is the following. 
In the general case the next conditions take place:

$$
\begin{array}{r}
\sup _{u(t, x, y, \varepsilon)} \inf _{\left(x_{1}(\cdot), y_{1}(\cdot)\right)} \inf _{s \in\left[t_{0},+\infty\right]} g_{A}\left(x_{1}(s), y_{1}(s)\right) \leq \\
\inf _{v(t, x, y, \varepsilon)} \sup _{\left(x_{2}(\cdot), y_{2}(\cdot)\right)} \inf _{s \in\left[t_{0},+\infty\right]} g_{A}\left(x_{2}(s), y_{2}(s)\right) \leq \\
\lim _{T \rightarrow+\infty} \min _{v(t, x, y, \varepsilon)} \max _{\left(x_{2}(\cdot), y_{2}(\cdot)\right)} \min _{s \in\left[t_{0}, T\right]} g_{A}\left(x_{2}(s), y_{2}(s)\right)= \\
\lim _{T \rightarrow+\infty} \max _{u(t, x, y, \varepsilon)} \min _{\left(x_{1}(\cdot), y_{1}(\cdot)\right)} \min _{s \in\left[t_{0}, T\right]} g_{A}\left(x_{1}(s), y_{1}(s)\right)=w_{A}(t, x, y) .
\end{array}
$$

One can verify the next properties of the function $w_{A}(t, x, y)$.

Property 1. The function $w_{A}$ is a stationary one

$$
w_{A}(t, x, y)=w_{A}(s, x, y)=w_{A}(x, y),\left(x_{i}, y_{i}\right) \in[0,1] \times[0,1], t \in \mathbb{R}, s \in \mathbb{R} .
$$

Property 2. The function $w_{A}$ satisfies the Lipschitz condition

$\left|w_{A}\left(x_{1}, y_{1}\right)-w_{A}\left(x_{2}, y_{2}\right)\right| \leq K\left(\left|x_{1}-x_{2}\right|+\left|y_{1}-y_{2}\right|\right), \quad\left(x_{i}, y_{i}\right) \in[0,1] \times[0,1], i=1,2$.

Property 3. The function $w_{A}$ is majorized by the payoff $g_{A}$

$$
w_{A}(x, y) \leq g_{A}(x, y), \quad(x, y) \in[0,1] \times[0,1] .
$$

Property 4. The function $w_{A}$ is the maximal function that satisfies the condition (16) and the principle of the dynamic programming. Namely, the properties of $u$ stability and $v$-stability can be presented as follows

$$
\begin{aligned}
& \min _{0 \leq v \leq 1} \max _{0 \leq u \leq 1} \partial_{+} w_{A}(x, y) \mid(-x+u,-y+v) \geq 0,(x, y) \in(0,1) \times(0,1), \\
& \max _{0 \leq u \leq 1} \min _{0 \leq v \leq 1} \partial_{-} w_{A}(x, y) \mid(-x+u,-y+v) \geq 0,(x, y) \in(0,1) \times(0,1) .
\end{aligned}
$$

Property 5. The properties of $u$-stability (17) and $v$-stability (18) can be rewritten in terms of the conjugate derivatives (Subbotin and Tarasyev, 1985)

$$
\begin{gathered}
D_{*} w_{A}(x, y) \mid(s) \leq H(x, y, s),(x, y) \in(0,1) \times(0,1), s=\left(s_{1}, s_{2}\right) \in \mathbb{R}^{2}, \\
D^{*} w_{A}(x, y) \mid(s) \geq H(x, y, s), \\
(x, y) \in(0,1) \times(0,1), w_{A}(x, y)<g_{A}(x, y), s=\left(s_{1}, s_{2}\right) \in \mathbb{R}^{2} .
\end{gathered}
$$

Taking into account the stability properties and using the concept of strategy of "extremal shift" (Krasovskii, 1985), one can prove that the corresponding trajectories provide that the value of the functional $G_{A}(14)$ in $\left[t_{0},+\infty\right)$ is equal to the value of the function $w_{A}$. Hence, all inequalities in (15) turn into the equalities, and this fact proves the Theorem.

\subsection{Description of Solution for Game with Multi-Terminal Functional}

For description of the analytic solution of the game let us introduce smooth components $\varphi_{1}(8), \varphi_{3}$ (10) of the value function $w_{1}(12)$ in terms of the backward time parameter $s=t-T$. Let us construct the lower envelopes of these smooth 
components, which present the multi-terminal interests of players. In constructing the envelope $\psi_{A}^{1}$ for the component $\varphi_{1}$ it is necessary to calculate the derivative by the parameter $s$, to equate it to zero, to find the root of the resulting equation, and to substitute the root in the component $\varphi_{1}$. Finally, we obtain

$$
\psi_{A}^{1}(x, y)=\varphi_{1}(s, x, y)=a_{22}-\frac{\left(\alpha_{1} x+\alpha_{2} y\right)^{2}}{4 C_{A} x y}
$$

Analogously, for the component $\varphi_{3}$ we obtain its lower envelope $\psi_{A}^{2}$ by the parameter $s$

$$
\psi_{A}^{2}(x, y)=\varphi_{3}(s, x, y)=a_{11}-\frac{\left(\left(C_{A}-\alpha_{1}\right)(1-x)+\left(C_{A}-\alpha_{2}\right)(1-y)\right)^{2}}{4 C_{A}(1-x)(1-y)} .
$$

In a similar way, we determine the lower envelopes $\psi_{A}^{3}, \psi_{A}^{4}$ of the components $\varphi_{2}$ $(9), \varphi_{4}(11)$

$$
\begin{aligned}
& \psi_{A}^{3}(x, y)=C_{A} x y-\alpha_{1} x-\alpha_{2} y+a_{22}, \\
& \psi_{A}^{4}(x, y)=\frac{a_{22} C_{A}-\alpha_{1} \alpha_{2}}{C_{A}}=\omega_{A} .
\end{aligned}
$$

The smooth functions $\psi_{A}^{i}, i=1, \ldots, 4$, are pasting on the lines $K_{A}^{j}, j=1, \ldots, 5$,

$$
\begin{aligned}
& K_{A}^{1}=\left\{(x, y): x=\frac{\alpha_{2}}{C_{A}}, 0 \leq y \leq 1\right\}, \\
& K_{A}^{2}=\left\{(x, y): \frac{\alpha_{2}}{C_{A}} \leq x \leq 1, \frac{\alpha_{1}}{C_{A}} \leq y \leq 1, y=\frac{\alpha_{1}}{\alpha_{2}} x\right\} \\
& K_{A}^{3}=\left\{(x, y): 0 \leq x \leq \frac{\alpha_{2}}{C_{A}}, 0 \leq y \leq \frac{\alpha_{1}}{C_{A}}, y=-\frac{\left(C_{A}-\alpha_{1}\right)}{\left(C_{A}-\alpha_{2}\right)}(1-x)+1\right\}, \\
& K_{A}^{4}=\left\{(x, y): \quad \frac{\alpha_{2}}{C_{A}} \leq x \leq 1,0 \leq y \leq \frac{\alpha_{1}}{C_{A}}, y=\frac{\alpha_{1} x}{2 C_{A} x-\alpha_{2}}\right\}, \\
& K_{A}^{5}=\left\{(x, y): 0 \leq x \leq \frac{\alpha_{2}}{C_{A}}, \frac{\alpha_{1}}{C_{A}} \leq y \leq 1, y=-\frac{\left(C_{A}-\alpha_{1}\right)(1-x)}{2 C_{A}(1-x)-\left(C_{A}-\alpha_{2}\right)}+1\right\},
\end{aligned}
$$

where $(x, y) \in[0,1] \times[0,1]$.

Let us introduce the analytic description of the value function $w_{A}$.

Statement 1. When $C_{A}>0$ the value function $(x, y) \mapsto w_{A}(x, y)$ is determined as

$$
w_{A}(x, y)=\psi_{A}^{i}(x, y), \quad \text { if } \quad(x, y) \in E_{A}^{i}, \quad i=1, \ldots, 4 .
$$

Here domains $E_{A}^{i}, i=1, \ldots, 4$, are given as follows 


$$
\begin{aligned}
E_{A}^{1}= & \left\{(x, y): \quad \frac{\alpha_{2}}{C_{A}} \leq x \leq 1, \quad \frac{\alpha_{1} x}{2 C_{A} x-\alpha_{2}} \leq y \leq \frac{\alpha_{1}}{\alpha_{2}} x\right\}, \\
E_{A}^{2}= & \left\{(x, y): \quad 0 \leq x \leq \frac{\alpha_{2}}{C_{A}},\right. \\
& \left.-\frac{\left(C_{A}-\alpha_{1}\right)}{\left(C_{A}-\alpha_{2}\right)}(1-x)+1 \leq y \leq-\frac{\left(C_{A}-\alpha_{1}\right)(1-x)}{2 C_{A}(1-x)-\left(C_{A}-\alpha_{2}\right)}+1\right\}, \\
E_{A}^{3}= & E_{A}^{31} \cup E_{A}^{32}, \\
E_{A}^{31}= & \left\{(x, y): \quad \frac{\alpha_{2}}{C_{A}} \leq x \leq 1, \quad 0 \leq y \leq \frac{\alpha_{1} x}{2 C_{A} x-\alpha_{2}}\right\}, \\
E_{A}^{32}= & \left\{(x, y): \quad 0 \leq x \leq \frac{\alpha_{2}}{C_{A}},\right. \\
& \left.-\frac{\left(C_{A}-\alpha_{1}\right)(1-x)}{2 C_{A}(1-x)-\left(C_{A}-\alpha_{2}\right)}+1 \leq y \leq 1\right\}, \\
E_{A}^{4}= & \left.E_{A}^{41} \cup E_{A}^{42}, \quad \alpha_{2} \leq x \leq 1, \quad \frac{\alpha_{1}}{\alpha_{2}} x \leq y \leq 1\right\}, \\
E_{A}^{41}= & \left\{(x, y): \quad \frac{\alpha_{A}}{C_{A}} \leq x \leq \frac{\left(C_{A}-\alpha_{1}\right)}{\left(C_{A}-\alpha_{2}\right)}(1-x)+1\right\}, \\
E_{A}^{42}= & \left\{(x, y): \quad 0 \leq x \leq \frac{\alpha_{2}}{C_{A}}, \quad 0 \leq y \leq\right.
\end{aligned}
$$

where $(x, y) \in[0,1] \times[0,1]$.

Let us consider, as an example, the following payoff matrix for the first player in the case when $C_{A}>0$ :

$$
A=\left(\begin{array}{ll}
3 & 1 \\
0 & 3
\end{array}\right)
$$

The game parameters for this matrix have the following values:

$$
C_{A}=5, \quad \alpha_{1}=2, \quad \alpha_{2}=3, \quad x_{A}=0.6, \quad y_{A}=0.4 .
$$

The structure of the value function $w_{A}$ for the matrix $A$ (23) in the case when $C_{A}>0$ is presented on Fig. 1.

Let us consider the case when $C_{A}<0$.

The lower envelopes in this case are determined as follows:

$$
\begin{aligned}
& \psi_{A}^{1}(x, y)=a_{21}+\frac{\left(\left(C_{A}-\alpha_{1}\right) x+\alpha_{2}(1-y)\right)^{2}}{4 C_{A} x(1-y)}, \\
& \psi_{A}^{2}(x, y)=a_{12}+\frac{\left(\alpha_{1}(1-x)+\left(C_{A}-\alpha_{2}\right) y\right)^{2}}{4 C_{A}(1-x) y}, \\
& \psi_{A}^{3}(x, y)=C_{A} x y-\alpha_{1} x-\alpha_{2} y+a_{22}, \\
& \psi_{A}^{4}(x, y)=\omega_{A} .
\end{aligned}
$$


Smooth functions $\psi_{A}^{i}, i=1, \ldots, 4$, are pasting on the lines $K_{A}^{j}, j=1, \ldots, 5$,

$$
\begin{aligned}
K_{A}^{1} & =\left\{(x, y): x=\frac{\alpha_{2}}{C_{A}}, 0 \leq y \leq 1\right\} \\
K_{A}^{2} & =\left\{(x, y): \quad \frac{\alpha_{2}}{C_{A}} \leq x \leq 1,0 \leq y \leq \frac{\alpha_{1}}{C_{A}}, y=-\frac{\left(C_{A}-\alpha_{1}\right)}{\alpha_{2}} x+1\right\}, \\
K_{A}^{3} & =\left\{(x, y): 0 \leq x \leq \frac{\alpha_{2}}{C_{A}}, \frac{\alpha_{1}}{C_{A}} \leq y \leq 1, y=\frac{\alpha_{1}}{\left(C_{A}-\alpha_{2}\right)}(1-x)\right\} \\
K_{A}^{4} & =\left\{(x, y): \frac{\alpha_{2}}{C_{A}} \leq x \leq 1, \quad \frac{\alpha_{1}}{C_{A}} \leq y \leq 1, y=-\frac{\left(C_{A}-\alpha_{1}\right) x}{2 C_{A} x-\alpha_{2}}+1\right\} \\
K_{A}^{5} & =\left\{(x, y): 0 \leq x \leq \frac{\alpha_{2}}{C_{A}}, 0 \leq y \leq \frac{\alpha_{1}}{C_{A}}, y=\frac{\alpha_{1}(1-x)}{2 C_{A}(1-x)-\left(C_{A}-\alpha_{2}\right)}+1\right\},
\end{aligned}
$$

where $(x, y) \in[0,1] \times[0,1]$.

The domains $E_{A}^{i}, i=1, \ldots, 4$, are determined as follows:

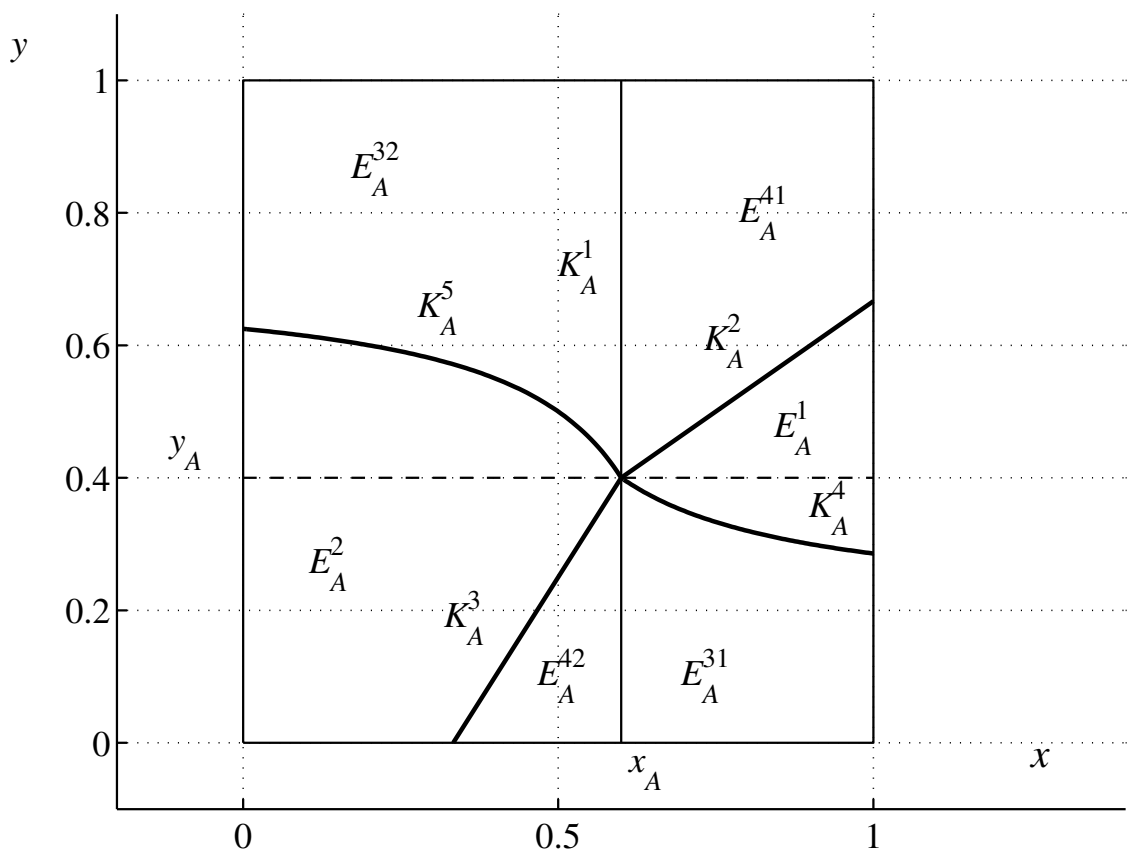

Fig. 1. The structure of the value function $w_{A}$ in the case when $C_{A}>0$. 


$$
\begin{aligned}
& E_{A}^{1}=\left\{(x, y): \quad \frac{\alpha_{2}}{C_{A}} \leq x \leq 1,\right. \\
& \left.-\frac{\left(C_{A}-\alpha_{1}\right)}{\alpha_{2}} x+1 \leq y \leq-\frac{\left(C_{A}-\alpha_{1}\right) x}{2 C_{A} x-\alpha_{2}}+1\right\}, \\
& E_{A}^{2}=\left\{(x, y): \quad 0 \leq x \leq \frac{\alpha_{2}}{C_{A}},\right. \\
& \left.\frac{\alpha_{1}(1-x)}{2 C_{A}(1-x)-\left(C_{A}-\alpha_{2}\right)}+1 \leq y \leq \frac{\alpha_{1}}{\left(C_{A}-\alpha_{2}\right)}(1-x)\right\}, \\
& E_{A}^{3}=E_{A}^{31} \cup E_{A}^{32}, \\
& E_{A}^{31}=\left\{(x, y): \quad \frac{\alpha_{2}}{C_{A}} \leq x \leq 1, \quad-\frac{\left(C_{A}-\alpha_{1}\right) x}{2 C_{A} x-\alpha_{2}}+1 \leq y \leq 1\right\}, \\
& E_{A}^{32}=\left\{(x, y): \quad 0 \leq x \leq \frac{\alpha_{2}}{C_{A}},\right. \\
& \left.0 \leq y \leq \frac{\alpha_{1}(1-x)}{2 C_{A}(1-x)-\left(C_{A}-\alpha_{2}\right)}+1\right\}, \\
& E_{A}^{4}=E_{A}^{41} \cup E_{A}^{42}, \\
& E_{A}^{41}=\left\{(x, y): \quad \frac{\alpha_{2}}{C_{A}} \leq x \leq 1, \quad 0 \leq y \leq-\frac{\left(C_{A}-\alpha_{1}\right)}{\alpha_{2}} x+1\right\}, \\
& E_{A}^{42}=\left\{(x, y): \quad 0 \leq x \leq \frac{\alpha_{2}}{C_{A}}, \quad \frac{\alpha_{1}}{\left(C_{A}-\alpha_{2}\right)}(1-x) \leq y \leq 1\right\},
\end{aligned}
$$

where $(x, y) \in[0,1] \times[0,1]$.

Let us consider, as an example, the following payoff matrix for the first player in the case when $C_{A}<0$ :

$$
A=\left(\begin{array}{cc}
-3 & -2 \\
0 & -4
\end{array}\right)
$$

The game parameters for this matrix have the following values:

$$
C_{A}=-5, \quad \alpha_{1}=-2, \quad \alpha_{2}=-4, \quad x_{A}=0.8, \quad y_{A}=0.4 .
$$

The structure of the value function $w_{A}$ for the matrix $A(25)$ in the case when $C_{A}<0$ is presented on Fig. 2.

Let us note that both cases of positive and negative signs of the basic parameter $C_{A}$, which generate different orientations, "left" and "right", for "zigzags" (broken lines) of acceptable situations in the static game (Vorobyev, 1985) is important for construction the Nash equilibrium trajectories in the considered below example of interactions of two players on the market of innovative electronic devices.

\subsection{Verification of $u$ - and $v$ - Stability in the Multi-Terminal Game}

In this section, we prove that the necessary and sufficient conditions for the function $w_{A}$ are satisfied for it coincidence with the value of a multi-terminal game.

Proposition 4. For the function $w_{A}$ in the case when $C_{A}>0$ the boundary condition (16) and differential inequalities (19), (20) are fulfilled.

Proof. The boundary condition obviously holds, because functions $\psi_{A}^{i}, i=1, \ldots, 4$ are the lower envelopes of the terminal solutions $w_{1}(T, t, x, y)$ and, hence,

$$
\psi_{A}^{i}(x, y) \leq \varphi_{i}(t, x, y) \leq g_{A}(x, y), \quad i=1, \ldots, 4, \quad(x, y) \in[0,1] \times[0,1] .
$$




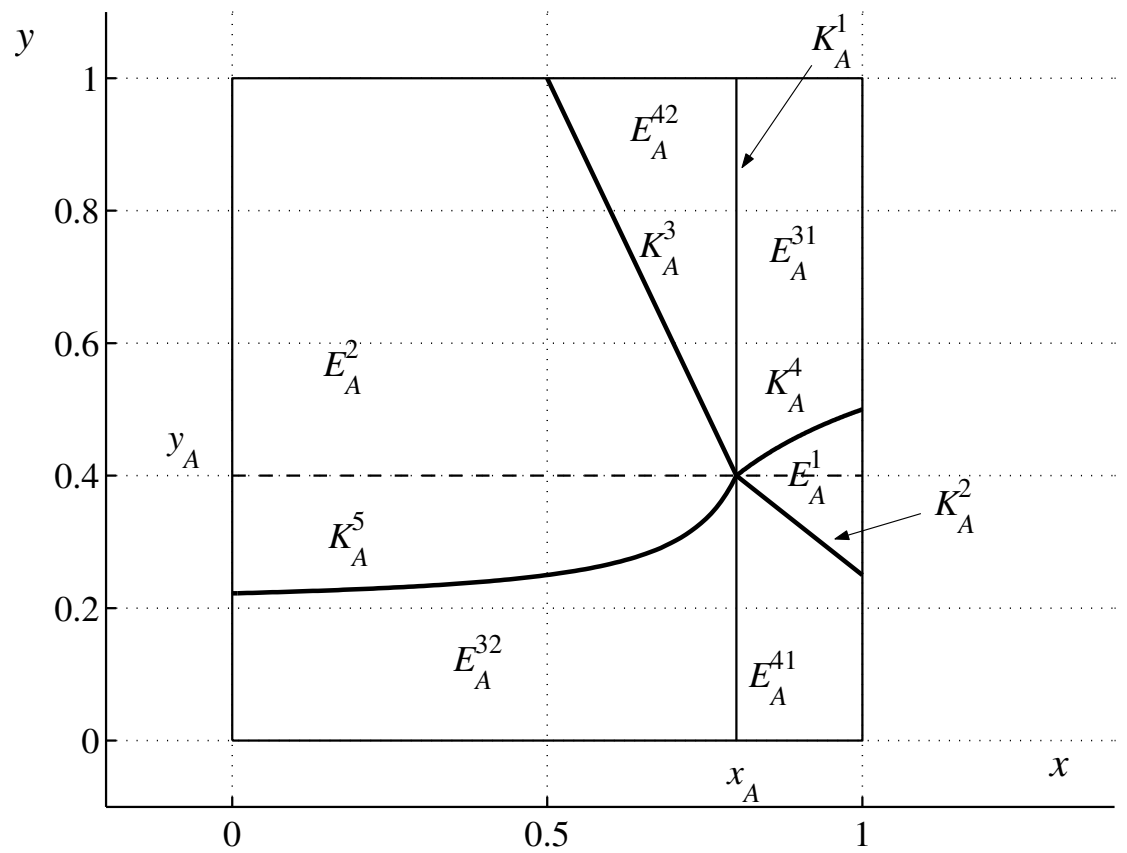

Fig. 2. The structure of the value function $w_{A}$ in the case when $C_{A}<0$.

Let us check, that the differential inequalities (19), (20) are fulfilled for the function $w_{A}$. One can prove that functions $\psi_{A}^{i}, i=1,2,4$, satisfy the HamiltonJacobi equation at the interior points of domains $E_{A}^{i}, i=1,2,4$. Also one can check, that the function $\psi_{A}^{3}(21)$ coincides with the boundary function $g_{A}$ and satisfies the inequality

$$
-\frac{\partial \psi_{A}^{3}}{\partial x} x-\frac{\partial \psi_{A}^{3}}{\partial y} y+\max \left\{0, \frac{\partial \psi_{A}^{3}}{\partial x}\right\}+\min \left\{0, \frac{\partial \psi_{A}^{3}}{\partial y}\right\} \geq 0
$$

at the interior points of the domain $E_{A}^{3}$.

It remains to verify the differential inequalities (19), (20) on the pasting lines $K_{A}^{j}, j=1, \ldots, 5$. Let us do this, for example, on the lines $K_{A}^{2}, K_{A}^{3}$. At the points of the line $K_{A}^{2}$, the functions $\psi_{A}^{1}$ and $\psi_{A}^{4}$ are continuously pasted. Let us calculate the partial derivatives of these functions

$$
\frac{\partial \psi_{A}^{1}}{\partial x}=\frac{\alpha_{2}^{2} y^{2}-\alpha_{1}^{2} x^{2}}{4 C_{A} x^{2} y}, \quad \frac{\partial \psi_{A}^{1}}{\partial y}=\frac{\alpha_{1}^{2} x^{2}-\alpha_{2}^{2} y^{2}}{4 C_{A} x y^{2}}, \quad \frac{\partial \psi_{A}^{4}}{\partial x}=0, \quad \frac{\partial \psi_{A}^{4}}{\partial y}=0 .
$$

One can note that these derivatives are equal to zero on the line $K_{A}^{2}$

$$
\frac{\partial \psi_{A}^{1}}{\partial x}=\frac{\partial \psi_{A}^{4}}{\partial x}=0, \quad \frac{\partial \psi_{A}^{1}}{\partial y}=\frac{\partial \psi_{A}^{4}}{\partial y}=0 .
$$

In other words, the functions $\psi_{A}^{1}$ and $\psi_{A}^{4}$ are smoothly pasted together. Hence, the inequalities (19), (20) on the line $K_{A}^{2}$ turn into the equality. Analogously, one can prove smooth pasting of the functions $\psi_{A}^{2}, \psi_{A}^{4}$ on the line $K_{A}^{3}$. 
Let us consider the line $K_{A}^{4}$, where the functions $\psi_{A}^{1}$ and $\psi_{A}^{3}$ are pasted together. One can check that this pasting is smooth, since for the partial derivatives on the line $K_{A}^{4}$ we obtain the relations

$$
\frac{\partial \psi_{A}^{1}}{\partial x}=\frac{\partial \psi_{A}^{3}}{\partial x}=\frac{\alpha_{1}\left(\alpha_{2}-C_{A} x\right)}{2 C_{A} x-\alpha_{2}}, \quad \frac{\partial \psi_{A}^{1}}{\partial y}=\frac{\partial \psi_{A}^{3}}{\partial y}=C_{A} x-\alpha_{2} .
$$

Similarly, one can check the smoothness of the function $w_{A}$ on the line $K_{A}^{5}$.

Along the line $K_{A}^{1}$ the functions $\psi_{A}^{3}$ and $\psi_{A}^{4}$ are pasted together. Their derivatives on the line $K_{A}^{1}$ are determined as

$$
\frac{\partial \psi_{A}^{3}}{\partial x}=C_{A} y-\alpha_{1}, \quad \frac{\partial \psi_{A}^{4}}{\partial x}=0, \quad \frac{\partial \psi_{A}^{3}}{\partial y}=\frac{\partial \psi_{A}^{4}}{\partial y}=0 .
$$

Let us note that there is no need to check the inequality (20) on the line $K_{A}^{1}$, since the following relations are fulfilled $w_{A}=\psi_{A}^{3}=\psi_{A}^{4}=g_{A}$. It is necessary only to verify the condition (19). One can check that in the vicinity of points of the line $K_{A}^{1}$ the function $w_{A}$ is determined by the operation of minimum

$$
w_{A}(x, y)=\min \left\{\psi_{A}^{3}(x, y), \psi_{A}^{4}(x, y)\right\} .
$$

Hence, for points $(x, y) \in K_{A}^{1}$ we obtain

$$
\begin{aligned}
\partial w_{A}(x, y) \mid\left(h_{1}, h_{2}\right) & =\min \left\{0,\left(C_{A} y-\alpha_{1}\right) h_{1}\right\}, \\
D_{*} w_{A}(x, y) \mid\left(s_{1}, s_{2}\right) & = \begin{cases}0, & s_{1}=\lambda\left(C_{A} y-\alpha_{1}\right), s_{2}=0, \\
-\infty, & \text { otherwise }\end{cases}
\end{aligned}
$$

Here the parameter $\lambda$ satisfies the inequalities $0 \leq \lambda \leq 1$. For points $(x, y) \in K_{A}^{1}$ and vectors $s=\left(s_{1}, s_{2}\right), s_{1}=\lambda\left(C_{A} y-\alpha_{1}\right), s_{2}=0$, the Hamiltonian $H(x, y, s)$ is determined by the relation

$$
H(x, y, s)=-s_{1} x+\max \left\{0, s_{1}\right\}= \begin{cases}-s_{1} x, & s_{1} \leq 0, \\ s_{1}(1-x), & \text { otherwise }\end{cases}
$$

It is obvious that for these values the Hamiltonian (27) is larger or equal to the lower conjugate derivative (26). Hence, the inequality (19) is proved on the line $K_{A}^{1}$.

Thus, we have proved that the function $w_{A}(21),(22)$ is the value function in the game with the multi-terminal functional.

The proof of the proposition (4) for the case when $C_{A}<0$ is carried out analogously.

Note 3. In the domain $E_{A}^{4}$, the next relations are valid

$$
g_{A}(x, y) \geq w_{A}(x, y)=\omega_{A} .
$$

Note 4. The positional strategy $U_{A}^{0}=u_{A}^{0}(x, y)$ corresponding to the value function $w_{A}$ (see relations $(28)$ ) provides the viability property for trajectories $(x(\cdot), y(\cdot))$ of the system (1) in the domain $E_{A}^{4}$.

Note 5 . For the matrix $B$ the value function $w_{B}$ and domains $E_{B}$ can be constructed analogously. 


\section{Flexible "Positive" Feedbacks}

\subsection{Optimal Feedback Controls}

Let us give the description of the flexible "positive" feedback controls $u_{A}^{0}=$ $u_{A}^{\mathrm{fl}}=u_{A}^{\mathrm{f}}(x, y)$, which solve the problem of guaranteeing maximization for the multiterminal functional $G_{A}\left(x_{1}(\cdot), y_{1}(\cdot)\right)$ (14) on trajectories $\left(x_{1}(\cdot), y_{1}(\cdot)\right)$ of the system (1). This control is constructed by the principle of the "extremal shift" in the direction of the gradient (generalized gradient) of the value function $w_{A}$.

Let us note that the partial derivative $\partial w_{A} / \partial x$ of the value function $w_{A}$ changes its sign on the lines $K_{A}^{2}$ and $K_{A}^{3}$. Thus, the optimal feedback control $u_{A}^{0}$ has the following structure (see, for example, (Krasovskii and Subbotin, 1988)). The control parameter $u_{A}^{\mathrm{f}}=u_{A}^{\mathrm{f}}(x, y)$ is equal to zero, if the current position $(x, y)=$ $\left(x_{1}(t), y_{1}(t)\right)$ lies on the right to the line $K_{A}=K_{A}^{2} \cup K_{A}^{3}$, equals to one, if the current position lies to the left of this line, and can take arbitrary values at points of the line $K_{A}$. Namely, if $C_{A}>0$, then

$$
u_{A}^{0}=u_{A}^{\mathrm{fl}}=u_{A}^{\mathrm{fl}}(x, y)= \begin{cases}0, & (x, y) \in D_{A}^{1}, \\ 1, & (x, y) \in D_{A}^{2}, \\ {[0,1],} & (x, y) \in K_{A} .\end{cases}
$$

$$
\begin{aligned}
D_{A}^{1} & =D_{A}^{11} \cup D_{A}^{12}, \\
D_{A}^{11} & =\left\{(x, y) \in[0,1] \times[0,1]: \quad y<\frac{\alpha_{1}}{\alpha_{2}} x, \quad y \geq \frac{\alpha_{1}}{C_{A}}\right\}, \\
D_{A}^{12} & =\left\{(x, y) \in[0,1] \times[0,1]: \quad y<-\frac{\left(C_{A}-\alpha_{1}\right)}{\left(C_{A}-\alpha_{2}\right)}(1-x)+1, \quad y \leq \frac{\alpha_{1}}{C_{A}}\right\}, \\
D_{A}^{2} & =D_{A}^{21} \cup D_{A}^{22}, \\
D_{A}^{21} & =\left\{(x, y) \in[0,1] \times[0,1]: \quad y>\frac{\alpha_{1}}{\alpha_{2}} x, \quad y \geq \frac{\alpha_{1}}{C_{A}}\right\}, \\
D_{A}^{22} & =\left\{(x, y) \in[0,1] \times[0,1]: \quad y>-\frac{\left(C_{A}-\alpha_{1}\right)}{\left(C_{A}-\alpha_{2}\right)}(1-x)+1, \quad y \leq \frac{\alpha_{1}}{C_{A}}\right\}, \\
K_{A} & =K_{A}^{2} \cup K_{A}^{3}, \\
K_{A}^{2} & =\left\{(x, y) \in[0,1] \times[0,1]: \quad y=\frac{\alpha_{1}}{\alpha_{2}} x, \quad y \geq \frac{\alpha_{1}}{C_{A}}\right\}, \\
K_{A}^{3} & =\left\{(x, y) \in[0,1] \times[0,1]: \quad y=-\frac{\left(C_{A}-\alpha_{1}\right)}{\left(C_{A}-\alpha_{2}\right)}(1-x)+1, \quad y \leq \frac{\alpha_{1}}{C_{A}}\right\} .
\end{aligned}
$$

If $C_{A}<0$, then the flexible "positive" feedback control $u_{A}^{\mathrm{fl}}$ has the analogous structure.

The guaranteeing problem for optimization of the multi-terminal functional $G_{B}\left(x_{2}(\cdot), y_{2}(\cdot)\right)$ for the second player is solved analogously.

\subsection{Multi-Terminal Optimal Control}

Optimal controls $u_{A}^{\mathrm{fl}}(x, y)(28)$ guarantee that the current payoff of the first player becomes in the long term not worse than the value $\omega_{A}=D_{A} / C_{A}$ of the matrix zero-sum game for the matrix $A$. The next statement is true. 
Statement 2. For any initial position $\left(x_{0}, y_{0}\right) \in[0,1] \times[0,1]$ and for any trajectory

$$
\left(x_{1}(\cdot), y_{1}(\cdot)\right) \in X\left(x_{0}, y_{0}, u_{A}^{\mathrm{f}}\right), \quad x_{1}\left(t_{0}\right)=x_{0}, \quad y_{1}\left(t_{0}\right)=y_{0}, \quad t_{0}=0,
$$

generated by the optimal control $u_{A}^{\mathrm{f}}=u_{A}^{\mathrm{f}}(x, y)$, there exists a finite time $t_{s} \in$ $\left[0, T_{A}\right]$,

$$
T_{A}=\ln \left(\max \left\{\frac{C_{A}}{\alpha_{2}}, \frac{C_{A}}{C_{A}-\alpha_{2}}\right\}\right),
$$

at which the trajectory $\left(x_{1}(\cdot), y_{1}(\cdot)\right)$ enters the domain $E_{A}^{4}$ (see $(22)$ ),

$$
\left(x_{1}\left(t_{s}\right), y_{1}\left(t_{s}\right)\right) \in E_{A}^{4},
$$

where the value function $w_{A}$ is equal to the value $\omega_{A}$ of the matrix game,

$$
w_{A}\left(x_{1}\left(t_{s}\right), y_{1}\left(t_{s}\right)\right)=\omega_{A},
$$

and stays in the domain $E_{A}^{4}$ on the time interval $\left[t_{s},+\infty\right.$ ) (and, hence, on the time interval $\left[T_{A},+\infty\right)$ ). Thus, according to the definition of the value function $w_{A}$, the next inequality takes place

$$
g_{A}\left(x_{1}(t), y_{1}(t)\right) \geq \omega_{A}, \quad t \geq t_{s}
$$

and, in particular,

$$
\liminf _{t \rightarrow+\infty} g_{A}\left(x_{1}(t), y_{1}(t)\right) \geq \omega_{A}
$$

Proof. Let us note that the domain $E_{A}^{4}$ (see (22)) has non-empty intersections with all lines $L_{\lambda}, 0 \leq \lambda \leq 1$,

$$
L_{\lambda}=\{(x, y) \in(0,1) \times(0,1): \quad y=\lambda\} .
$$

Hence, any trajectory $\left(x_{1}(\cdot), y_{1}(\cdot)\right)$, generated by the feedback optimal control $u_{A}^{\mathrm{f}}$ (the value of which is equal to zero or one) intersects this domain $E_{A}^{4}$. Thus, the projection of the velocity on the lines $L_{\lambda}$ for such trajectories is not equal to zero and keeps the sign till the moment of the intersection of the trajectory with the domain $E_{A}^{4}$.

The analogous statement can be formulated for the game with the matrix $B$.

Statement 3. Intersection $E^{0}$ of the sets $E_{A}^{4}$ and $E_{B}^{4}$ is non-empty, i.e. $E^{0}=$ $E_{A}^{4} \cap E_{B}^{4} \neq \emptyset$, and, hence, the optimal strategies $u_{A}^{\mathrm{f}}, v_{B}^{\mathrm{f}}$ generate the trajectory $\left(x^{\mathrm{H}}(\cdot), y^{\mathrm{H}}(\cdot)\right)$, which enters the intersection $E^{0}$ and stays in it on the time interval $\left[T^{0},+\infty\right), T^{0}=\max \left\{T_{A}, T_{B}\right\}$. In the set $E^{0}$, the following inequalities are fulfilled

$$
g_{A}\left(x^{\mathrm{fl}}(t), y^{\mathrm{fl}}(t)\right) \geq \omega_{A}, \quad g_{B}\left(x^{\mathrm{fl}}(t), y\left({ }^{\mathrm{f}} t\right)\right) \geq \omega_{B}, \quad t \in\left[T^{0},+\infty\right) .
$$

Thus, the set $E^{0}$ can be called the favorable domain for both players. 


\section{Nash Equilibrium with Flexible "Positive" Controls}

\subsection{Structure of the Nash Equilibrium}

Let us construct the pair of controls for the Nash equilibrium, pasting together the flexible "positive" controls $u_{A}^{0}=u_{A}^{\mathrm{f}}, v_{B}^{0}=v_{B}^{\mathrm{f}}$ and the "punishing" controls $u_{B}^{0}=u_{B}^{\mathrm{cl}}, v_{A}^{0}=v_{A}^{\mathrm{cl}}$. Let us choose the initial position $\left(x_{0}, y_{0}\right) \in[0,1] \times$ $[0,1]$ and the accuracy parameter $\varepsilon>0$. Let us fix the trajectory $\left(x^{\mathrm{f}}(\cdot), y^{\mathrm{f}}(\cdot)\right) \in$ $X\left(x_{0}, y_{0}, u_{A}^{\mathrm{f}}(\cdot), v_{B}^{\mathrm{f}}(\cdot)\right)$, generated by the flexible "positive" controls $u_{A}^{\mathrm{f}}$ and $v_{B}^{\mathrm{f}}$. Let us choose the moment of time $T_{\varepsilon}>0$ such that

$$
\begin{gathered}
g_{A}\left(x^{\mathrm{f}}(t), y^{\mathrm{f}}(t)\right)>J_{A}^{-}\left(x^{\mathrm{f}}(\cdot), y^{\mathrm{f}}(\cdot)\right)-\varepsilon, \\
g_{B}\left(x^{\mathrm{f}}(t), y^{\mathrm{f}}(t)\right)>J_{B}^{-}\left(x^{\mathrm{f}}(\cdot), y^{\mathrm{f}}(\cdot)\right)-\varepsilon, \\
t \in\left[T_{\varepsilon},+\infty\right) .
\end{gathered}
$$

Let us denote by the symbol $u_{A}^{f l, \varepsilon}(t):\left[0, T_{\varepsilon}\right) \rightarrow[0,1], v_{B}^{f l, \varepsilon}(t):\left[0, T_{\varepsilon}\right) \rightarrow[0,1]$, the step-by-step realization of the strategies $u_{A}^{\mathrm{f}}, v_{B}^{\mathrm{fl}}$, such that the corresponding step-by step motion $\left(x_{\varepsilon}^{\mathrm{f}}(\cdot), y_{\varepsilon}^{\mathrm{fl}}(\cdot)\right)$ satisfies the condition

$$
\max _{t \in\left[0, T_{\varepsilon}\right]}\left\|\left(x^{\mathrm{f}}(t), y^{\mathrm{f}}(t)\right)-\left(x_{\varepsilon}^{\mathrm{f}}(t), y_{\varepsilon}^{\mathrm{f}}(t)\right)\right\|<\varepsilon .
$$

Applying the construction of the Nash equilibrium from (Kleimenov, 1993), we obtain the following result.

Statement 4. The pair of controls $U^{0}=u^{0}(t, x, y, \varepsilon), V^{0}=v^{0}(t, x, y, \varepsilon)$ pasted together by the flexible "positive" controls $u_{A}^{\mathrm{fl}}, v_{B}^{\mathrm{f}}$, and the "punishing" controls $u_{B}^{\mathrm{cl}}$, $v_{A}^{\mathrm{cl}}$,

$$
\begin{aligned}
& U^{0}=u^{0}(t, x, y, \varepsilon)= \begin{cases}u_{A}^{f l, \varepsilon}(t), \quad\left\|(x, y)-\left(x_{\varepsilon}^{\mathrm{f}}(t), y_{\varepsilon}^{\mathrm{fl}}(t)\right)\right\|<\varepsilon, \\
u_{B}^{\mathrm{cl}}(x, y), \text { otherwise }\end{cases} \\
& V^{0}=v^{0}(t, x, y, \varepsilon)=\left\{\begin{array}{l}
v_{B}^{f l, \varepsilon}(t),\left\|(x, y)-\left(x_{\varepsilon}^{\mathrm{fl}}(t), y_{\varepsilon}^{\mathrm{fl}}(t)\right)\right\|<\varepsilon, \\
v_{A}^{\mathrm{cl}}(x, y), \text { otherwise }
\end{array}\right.
\end{aligned}
$$

is the dynamic $\varepsilon$-Nash equilibrium.

Let us note that the trajectory $\left(x_{\varepsilon}^{\mathrm{f}}(\cdot), y_{\varepsilon}^{\mathrm{f}}(\cdot)\right)$ is the core of the dynamic Nash equilibrium. Thus, it can be called the equilibrium trajectory. It is generated by the guaranteeing controls $u_{A}^{\mathrm{fl}}$ and $v_{B}^{\mathrm{f}}$, and provides values for both payoff functionals better than the static Nash equilibrium.

\subsection{Trajectories for Felxible "Positive" Controls}

The question of interest is the qualitative behavior of the trajectories generated by the flexible "positive" controls which form the basis for the dynamic Nash equilibrium (29), (30). To classify the possible behavior of these trajectories, we can formulate the following statement.

Statement 5. The values of the payoff functionals $J_{A}^{-}, J_{B}^{-}$on the arbitrary trajectory $\left(x^{\mathrm{f}}(\cdot), y^{\mathrm{f}}(\cdot)\right)$ generated by the flexible "positive" feedback controls $u_{A}^{\mathrm{f}}$, $v_{B}^{\mathrm{fl}}$, are not worse than values of these functionals on any trajectory converging to the static Nash equilibrium $\left(x_{B}, y_{A}\right)=\left(\beta_{2} / C_{B}, \alpha_{1} / C_{A}\right)$, on which the components of players' distributions are unfavorable for opposite players. Moreover, the 
trajectories $\left(x^{\mathrm{f}}(\cdot), y^{\mathrm{f}}(\cdot)\right)$ enter the favorable domain $E^{0}$ and stay there on the infinite time interval. The next variants of the qualitative behavior of the trajectories $\left(x^{\mathrm{f}}(\cdot), y^{\mathrm{f}}(\cdot)\right)$ in the domain $E^{0}$ are possible:

- it can converge to the intersection of lines $K_{A}, K_{B}$;

- it can approach the points located on the border of the square (in the case when the intersection of lines $K_{A}, K_{B}$ is empty);

- it can approach the non-antagonistic static Nash equilibrium with Pareto properties (in the case when such equilibrium exists);

- it can circulate in the domain $E^{0}$.

\section{Examples of Equilibrium Trajectories in Evolutionary Bimatrix Games}

Let us consider, as an example, the payoff matrices of two players on the market of electronics: Samsung Corporation (matrix $A$ ) and Apple Corporation (matrix $B)$ :

$$
A=\left(\begin{array}{ll}
a_{11} & a_{12} \\
a_{21} & a_{22}
\end{array}\right), \quad B=\left(\begin{array}{ll}
b_{11} & b_{12} \\
b_{21} & b_{22}
\end{array}\right) .
$$

We consider annual sales of players and their investments in 2018 according to the data from the internet. Resources that we used are the following:

https://news.samsung.com/

http://www.annualreports.com/Company/apple-inc

Let us present the elements of matrices $A$ and $B$ :

$$
\begin{aligned}
a_{11} & =\left(S_{o}^{1}+\left(S_{b}^{1}+S_{b}^{2}\right) / 2-I_{l}^{1}\right), \\
a_{12} & =\left(S_{o}^{1}+S_{b}^{1}-I_{l}^{1}\right), \\
a_{21} & =\left(S_{o}^{1}+S_{a}^{1}-I_{s}^{1}\right), \\
a_{22} & =\left(S_{o}^{1}-I_{s}^{1}\right) . \\
b_{11} & =\left(S_{o}^{2}+\left(S_{b}^{1}+S_{b}^{2}+S_{a}^{2}\right) / 3-I_{l}^{2}\right), \\
b_{12} & =\left(S_{o}^{2}+S_{b}^{2}-I_{l}^{2}\right), \\
b_{21} & =\left(S_{o}^{2}+S_{a}^{2}-I_{s}^{2}\right), \\
b_{22} & =\left(S_{o}^{2}-I_{s}^{2}\right) .
\end{aligned}
$$

We obtain payoff matrices in the view:

$$
\begin{aligned}
& A=\left(\begin{array}{cc}
\left(S_{o}^{1}+\left(S_{b}^{1}+S_{b}^{2}\right) / 2-I_{l}^{1}\right) & \left(S_{o}^{1}+S_{b}^{1}-I_{l}^{1}\right) \\
\left(S_{o}^{1}+S_{a}^{1}-I_{s}^{1}\right) & \left(S_{o}^{1}-I_{s}^{1}\right)
\end{array}\right), \\
& B=\left(\begin{array}{cc}
\left(S_{o}^{2}+\left(S_{b}^{1}+S_{b}^{2}+S_{a}^{2}\right) / 3-I_{l}^{2}\right) & \left(S_{o}^{2}+S_{b}^{2}-I_{l}^{2}\right) \\
\left(S_{o}^{2}+S_{a}^{2}-I_{s}^{2}\right) & \left(S_{o}^{2}-I_{s}^{2}\right)
\end{array}\right) .
\end{aligned}
$$

Here $S_{o}^{1}=220$ billion dollars is the ordinary sales of Samsung corp.;

$S_{b}^{1}=22$ billion dollars is the bonus sales of Samsung corp. due to investments in new technologies;

$S_{a}^{1}=8$ billion dollars is the sales of Samsung corp. due to absorption of new technologies from Apple corp. without own investments;

$I_{l}^{1}=15$ billion dollars is the long-term investments of Samsung corp.;

$I_{s}^{1}=0$ billion dollars is the short-term investments of Samsung corp.;

$S_{o}^{2}=215$ billion dollars is the ordinary sales of Apple corp.;

$S_{b}^{2}=14$ billion dollars is the bonus sales of Apple corp. due to investments in new 
technologies;

$S_{a}^{2}=4$ billion dollars is the sales of Apple corp. due to absorption of new technologies from Samsung corp. without own investments;

$I_{l}^{2}=11.5$ billion dollars is the long-term investments of Apple corp.;

$I_{s}^{2}=0$ billion dollars is the short-term investments of Apple corp.;

The resulting payoff matrices have the following form:

$$
A=\left(\begin{array}{ll}
223 & 227 \\
228 & 220
\end{array}\right), \quad B=\left(\begin{array}{cc}
216.83 & 217.5 \\
219 & 215
\end{array}\right)
$$

The main game parameters are determined as follows:

$$
\begin{gathered}
C_{A}=a_{11}-a_{12}-a_{21}+a_{22}=-12, \\
\alpha_{1}=a_{22}-a_{12}=-7, \quad \alpha_{2}=a_{22}-a_{21}=-8, \\
x_{A}=\frac{\alpha_{2}}{C_{A}}=0.67, \quad y_{A}=\frac{\alpha_{1}}{C_{A}}=0.58 . \\
C_{B}=b_{11}-b_{12}-b_{21}+b_{22}=-4.67, \\
\beta_{1}=b_{22}-b_{12}=-2.5, \quad \beta_{2}=b_{22}-b_{21}=-4, \\
x_{B}=\frac{\beta_{2}}{C_{B}}=0.86, \quad y_{B}=\frac{\beta_{1}}{C_{B}}=0.54 .
\end{gathered}
$$

On Fig. 3 we present the saddle point $S_{A}$ of the static game with the matrix $A$, the switching line $K_{A}$, and the vector field of motions for the first player.

On Fig. 4 we present the saddle point $S_{B}$ of the static game with the matrix $B$, the switching line $K_{B}$, and the vector field of motions for the second player.

On Fig. 5 we present three situations of the Nash equilibrium $N_{1}, N_{2}$ and $N_{3}$ in the static game; the switching lines $K_{A}$ and $K_{B}$; the equilibrium trajectories $T_{1}$,

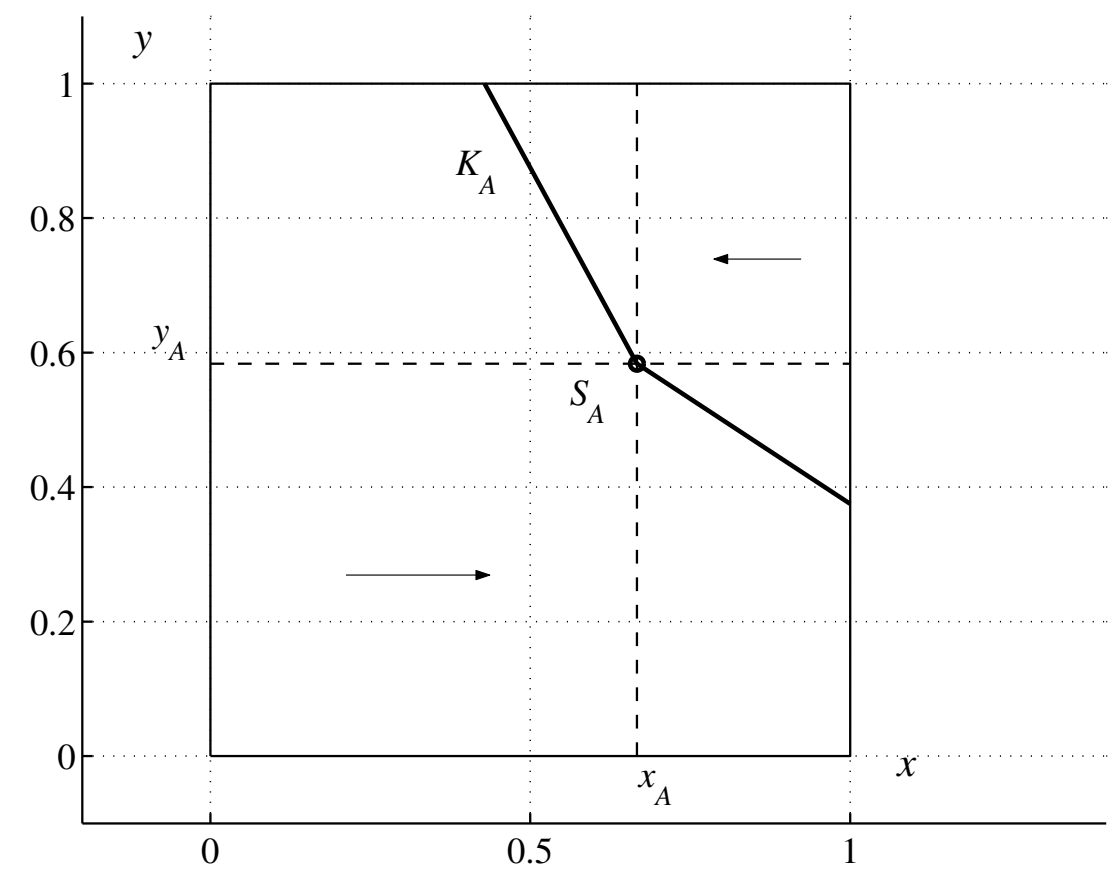

Fig. 3. Switching lines for the control feedback of the first player. 


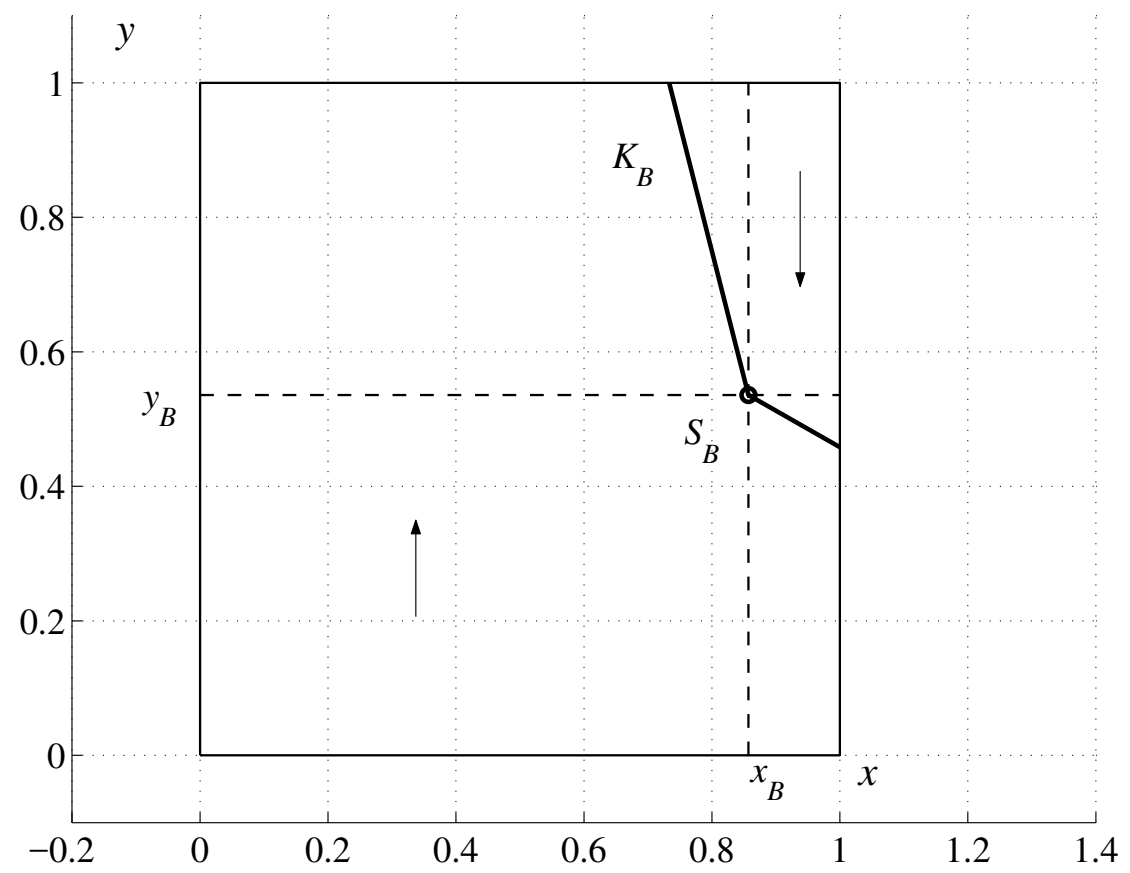

Fig. 4. Switching lines for the control feedback of the second player.

$T_{2}$ and $T_{3}$ starting from the initial points $I P_{1}, I P_{2}$ and $I P_{3}$ and converging to the point $F_{1}$ of the Pareto maximum.

Values of the functionals $g_{A}$ and $g_{B}$ at the final point $F_{1}$ of the equilibrium trajectories are better than at the points of the Nash equilibrium $N_{1}$ and $N_{2}$. Values of the functionals $g_{A}$ and $g_{B}$ at the point $N_{3}$ are better than at the point $F_{1}$, which can be explained as follows. Both players are aimed to approach this point, but the first player insures against big payoff losses and stays in the favorable domain. So, at the limit point of the Nash equilibrium trajectory the first player prefers to share investments between own technologies and absorption of the opponent technologies, and the second player makes the decision to rely fully on own technologies.

\section{Conclusion}

The paper deals with development of the generalized method of characteristics for construction of the generalized minimax (viscosity) solutions of Hamilton-Jacobi equations in dynamic bimatrix games. These solutions play the key role in the structure of the dynamic Nash equilibrium since they synthesize optimal feedbacks of players and generate equilibrium trajectories with the guaranteeing properties. We obtain the analytical formulas for the value functions as the generalized minimax (viscosity) solutions of the Hamilton-Jacobi equations in the cases of different orientations for the "zigzags" (broken lines) of acceptable situations in the static game. It is proved that the equilibrium trajectories generated by the generalized minimax solutions shift the system in the direction of cooperative Pareto points. The proposed approach provides better results of payoffs for both players at the limit points 


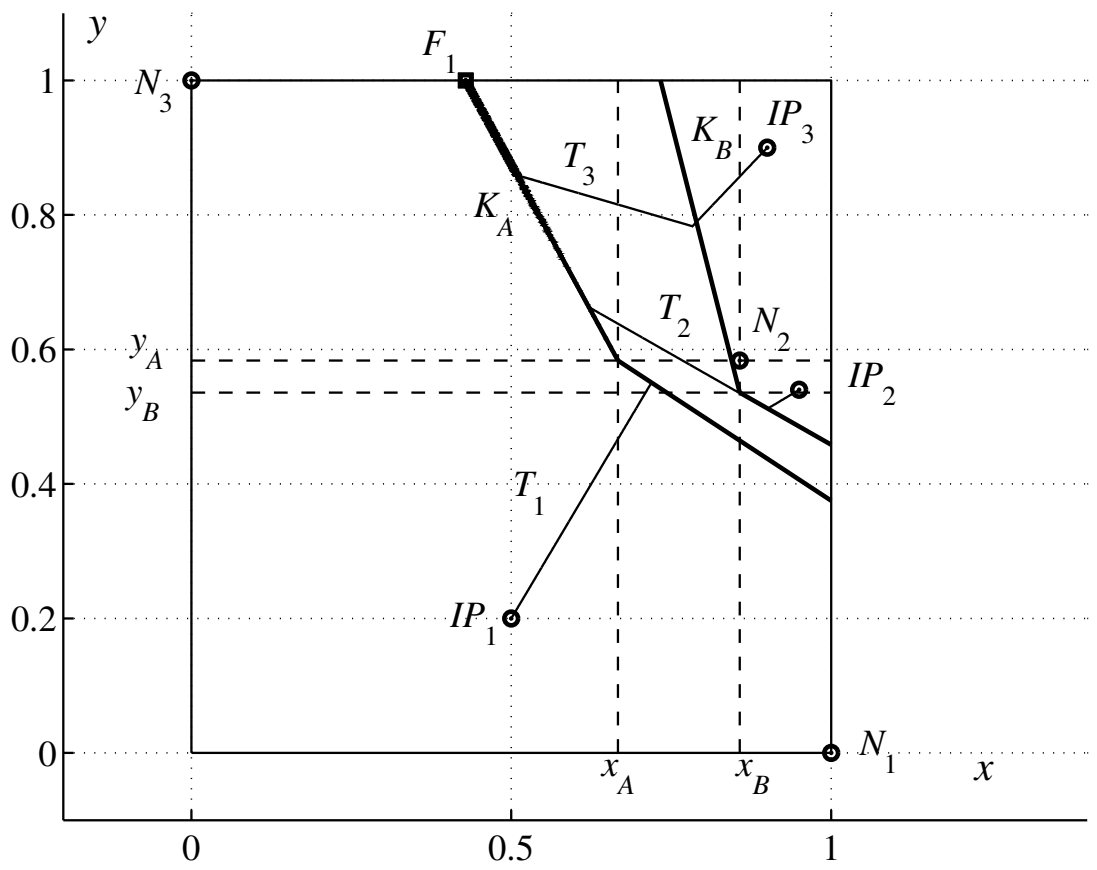

Fig. 5. Equilibrium trajectories of the dynamic bimatrix game.

of the equilibrium trajectories in the dynamic bimatrix games than the static Nash equilibrium or trajectories of the replicator dynamics in the evolutionary games. We consider an example of two competitive players on the market of innovative electronic devices and construct the Nash equilibrium trajectories in the dynamic bimatrix games which shift unfavorable Nash equilibria to the set of Pareto maximum points.

\section{References}

Aubin, J. P. (1990). A survey of viability theory. SIAM J. Control Optim., 28(4), 749-788.

Basar, T. and Olsder, G.J. (1982). Dynamic Noncooperative Game Theory. Academic: London.

Crandall, M. G. and Lions, P. L. (1983). Viscosity solutions of Hamilton-Jacobi equations. Trans. Amer. Math. Soc., 277(4), 1-42.

Friedman, D. (1991). Evolutionary games in economics. Econometrica, 59(3), 637-666.

Hofbauer, J. and Sigmund, K. (1988). The Theory of Evolution and Dynamic Systems. Cambridge Univ. Press: Cambridge.

Intriligator, M. (1971). Mathematical Optimization and Economic Theory. Prentice-Hall: New York.

Klaassen, G. Kryazhimskii, A. V. and Tarasyev, A. M. (2004). Multiequilibrium game of timing and competition of gas pipeline projects. Journal of Optimization Theory and Applications, 120(1), 147-179.

Kleimenov, A.F. (1993). Nonantagonistic Positional Differential Games. Nauka: Yekaterinburg.

Krasovskii, A. A. and Tarasyev, A. M. (2008). Conjugation of Hamiltonian systems in optimal control problems. IFAC Proceedings Volumes, 41(2), 7784-7789. 
Krasovskii, A. N. and Krasovskii, N. N. (1995). Control Under Lack of Information. Birkhauser: Boston etc.

Krasovskii, N. A. and Tarasyev, A. M. (2011). Decomposition algorithm of searching equilibrium in a dynamic game. Mathematical Game Theory and Applications, 3(4), 49-88.

Krasovskii, N. A. and Tarasyev, A. M. (2015). Equilibrium Solutions in Dynamic Games. UrGAU: Yekaterinburg.

Krasovskii, N. A. and Tarasyev, A. M. (2018). Equilibrium trajectories in dynamical bimatrix games with average integral payoff functionals. Automation and Remote Control, $\mathbf{7 9}(6), 1148-1167$.

Krasovskii, N. N. (1985). Control of Dynamic System. Nauka: Moscow.

Krasovskii, N. N. and Subbotin, A. I. (1988). Game-Theoretical Control Problems. Springer: New York.

Kryazhimskii, A. V. Osipov, Yu. S. (1995). On differential-evolutionary games. Proceedings of the Steklov Institute of Mathematics, 211, 234-261.

Kurzhanskii, A. B. (1977). Control and Observation Under Uncertainty. Nauka: Moscow.

Petrosjan, L. A. and Zenkevich, N. A. (2015). Conditions for sustainable cooperation. Automation and Remote Control, 76(10), 1894-1904.

Pontryagin, L.S. Boltyanskii, V. G. Gamkrelidze, R. V. and Mischenko, E. F. (1961). The Mathematical Theory of Optimal Processes. Interscience Publishers: New York.

Subbotin, A.I. (1991). Minimax Inequalities and Hamilton-Jacobi Equations. Nauka: Moscow.

Subbotin, A. I. and Tarasyev, A. M. (1985). Conjugate derivatives of the value function of a differential game. Doklady AN SSSR 283(3), 559-564.

Tarasyev, A.M. (1994). Approximation schemes for constructing minimax solutions of Hamilton-Jacobi equations. Journal of Applied Mathematics and Mechanics, 58(2), $207-221$.

Tarasyev, A. M. (1994). A Differential model for a $2 \times 2$-evolutionary game dynamics. IIASA working paper, Laxenburg, Austria, WP-94-063.

Tarasyev, A. M. and Watanabe, C. (2001). Dynamic optimality principles and sensitivity analysis in models of economic growth. Nonlinear Analysis, 47(4), 2309-2320.

Vorobyev, N. N. (1985). Game Theory for Economists and System Scientists. Nauka: Moscow. 